\title{
GAS KINEMATICS ON GIANT MOLECULAR CLOUD SCALES IN M51 WITH PAWS: CLOUD STABILIZATION THROUGH DYNAMICAL PRESSURE
}

\author{
Sharon E. Meidt ${ }^{1}$, Eva Schinnerer ${ }^{1}$, Santiago García-Burillo ${ }^{2}$, Annie Hughes ${ }^{1}$, Dario Colombo ${ }^{1}$, Jérôme Pety ${ }^{3,4}$, \\ Clare L. Dobbs $^{5}$, Karl F. Schuster ${ }^{3}$, Carsten Kramer $^{6}$, Adam K. Leroy ${ }^{7}$, Galle Dumas $^{3}$, and Todd A. Thompson ${ }^{8,9}$ \\ ${ }^{1}$ Max-Planck-Institut für Astronomie/Königstuhl 17 D-69117 Heidelberg, Germany \\ ${ }^{2}$ Observatorio Astronómico Nacional - OAN, Observatorio de Madrid Alfonso XII, 3, E-28014 - Madrid, Spain \\ ${ }^{3}$ Institut de Radioastronomie Millimétrique, 300 Rue de la Piscine, F-38406 Saint Martin d'Hères, France \\ ${ }^{4}$ Observatoire de Paris, 61 Avenue de l'Observatoire, F-75014 Paris, France \\ ${ }^{5}$ School of Physics and Astronomy, University of Exeter, Stocker Road, Exeter EX4 4QL, UK \\ ${ }^{6}$ Instituto Radioastronomía Milimétrica, Avenida Divina Pastora 7, Nucleo Central, E-18012 Granada, Spain \\ ${ }^{7}$ National Radio Astronomy Observatory, 520 Edgemont Road, Charlottesville, VA 22903, USA \\ ${ }^{8}$ Department of Astronomy, The Ohio State University, 140 West 18th Avenue, Columbus, OH 43210, USA \\ ${ }^{9}$ Center for Cosmology and AstroParticle Physics, The Ohio State University, 191 West Woodruff Avenue, Columbus, OH 43210, USA \\ Received 2012 October 31; accepted 2013 April 29; published 2013 November 25
}

\begin{abstract}
We use the high spatial and spectral resolution of the PAWS CO(1-0) survey of the inner $9 \mathrm{kpc}$ of the iconic spiral galaxy M51 to examine the effects of gas streaming motions on the star-forming properties of individual giant molecular clouds (GMCs). We compare our view of gas flows in M51-which arise due to departures from axisymmetry in the gravitational potential (i.e., the nuclear bar and spiral arms) - with the global pattern of star formation as traced by $\mathrm{H} \alpha$ and $24 \mu \mathrm{m}$ emission. We find that the dynamical environment of GMCs strongly affects their ability to form stars, in the sense that GMCs situated in regions with large streaming motions can be stabilized, while similarly massive GMCs in regions without streaming go on to efficiently form stars. We argue that this is the result of reduced surface pressure felt by clouds embedded in an ambient medium undergoing large streaming motions, which prevent collapse. Indeed, the variation in gas depletion time expected based on the observed streaming motions throughout the disk of M51 quantitatively agrees with the variation in the observed gas depletion time scale. The example of M51 shows that streaming motions, triggered by gravitational instabilities in the form of bars and spiral arms, can alter the star formation law; this can explain the variation in gas depletion time among galaxies with different masses and morphologies. In particular, we can explain the long gas depletion times in spiral galaxies compared with dwarf galaxies and starbursts. We suggest that adding a dynamical pressure term to the canonical free-fall time produces a single star formation law that can be applied to all star-forming regions and galaxies across cosmic time.
\end{abstract}

Key words: galaxies: individual (M51) - galaxies: ISM - galaxies: star formation - ISM: kinematics and dynamics

\section{INTRODUCTION}

Our understanding of how galaxies build up their massfundamental to our picture of galaxy evolution-relies heavily on the relation between newborn stars and the dense molecular material out of which they form. At present, the empirical Kennicutt-Schmidt (KS; Schmidt 1959; Kennicutt 1989) relation linking the star formation rate (SFR) surface density $\Sigma_{\mathrm{SFR}}$ with a power-law dependence on gas surface density $\Sigma_{\text {gas }}$ is in wide use by numerical simulations that inform our view of galaxy evolution. This relation has been demonstrated to hold over several orders of magnitude in $\Sigma_{\text {gas }}$ and across a range of galaxy types. The first homogeneous, spatially resolved observations in nearby galaxies (e.g., Bigiel et al. 2008; Leroy et al. $2008,2009,2012$ ) suggest that gas is consumed in star formation (SF) at a fixed efficiency, leading to an apparently "universal," but long, gas depletion time of $\sim 2.5 \mathrm{Gyr}$ for galaxies. The relation was further shown to be determined predominantly by the molecular phase of the neutral gas (Schruba et al. 2011; Bigiel et al. 2008).

At the same time, there is mounting evidence that SF may occur in two modes: the "normal" mode typical of the majority of SF in local disks and a "starburst" mode, wherein gas is consumed much more efficiently and quickly (i.e., Kennicutt 1998). Bimodality in the SF relation is manifested both locally (i.e., Leroy et al. 2013; Saintonge et al. 2012) and at higher $z$ (i.e., Daddi et al. 2010; Genzel et al. 2010; Garcia-Burillo et al. 2012). But, to date, there is no single "universal" SF relation than can smoothly link the two regimes across all times (but see Krumholz et al. 2012).

How the star-forming gas is organized may influence the depletion time (Combes et al. 2013; Saintonge et al. 2012 and references therein). An interpretation of the long depletion time in "normal" star-forming galaxies could be that it reflects only the (low) fraction of star-forming clouds rather than the fraction of a cloud that forms stars. Variations in the properties of giant molecular clouds (GMCs) themselves, the actual seeds of SF, could profoundly impact their SF capabilities, as evidenced by the large scatter of observed SFRs in local Galactic GMCs (Mooney \& Solomon 1988; Kennicutt \& Evans 2012; Heiderman et al. 2010). Yet, our understanding of the molecular gas phase and its organization into self-gravitating entities, i.e., GMCs, is limited (for a review, see McKee \& Ostriker 2007).

Observations of molecular gas at high resolution in nearby spiral galaxies are critical to test the emerging picture of how galactic environment influences the organization, properties, and evolutionary pathways of a galaxy's GMC population and its global patterns of SF. In this paper, we continue our study of the interacting Whirlpool galaxy M51 $(D=7.6 \mathrm{Mpc}$; Ciardullo et al. 2002), which is more representative of a typical star forming galaxy than the well-studied lower-mass galaxies in the Local Group. The unparalleled high resolution of the PdBI 
Arcsecond Whirlpool Survey (PAWS; Schinnerer et al. 2013) data, combined with exceptional multi-wavelength coverage, makes them an ideal target for examining the influence of bar and spiral instabilities - and more generally, the dynamical galactic environment - on the organization of the interstellar medium (ISM) and global SF patterns.

Our detailed analysis of the molecular gas in M51-including its GMC population - so far suggests that several mechanisms control its structure and evolution (Colombo et al. 2013a). Deviations in the gravitational potential from axisymmetry (i.e., the nuclear bar and two-armed spiral) induce shear, shocks, and streaming motions in the gas flow, leading to changes in the local gas surface density. This subsequently alters the pattern of radiation from recent $\mathrm{SF}$, which occurs on the convex side of the spiral arm, and therefore also the mechanical energy input from newly formed OB stars, stellar winds, and supernovae. The galactic environment is thus very dynamic in both senses and it is not clear under which conditions the developed static models apply (e.g., Krumholz et al. 2009).

Gas kinematics on the scales of individual GMCs are key for linking ongoing SF with the organization and radial transport of gas by the stellar spiral arms. We expect the dynamical environment to strongly impact the organization of the ISM in a galaxy like M51, which hosts strong, well-organized spiral structure and is presently undergoing an interaction with a companion. Depending on location in the disk, gas will be driven radially in- or outward and may occasionally be halted along its path, building up rings (see the review by Buta \& Combes 1996). Whether gas is in motion or stationary will naturally influence the build-up of the star-forming gas reservoir, the accumulation of mass into clouds, and the eventual collapse to form stars.

To quantify the influence of gas kinematics on cloud scales, we undertake a study in two parts. We begin by evaluating the close link between the gas depletion time $\tau_{\text {dep }}=\Sigma_{\text {gas }} /$ $\Sigma_{\mathrm{SFR}}{ }^{10}$ and dynamical environment as defined by the radial profile of present-day gravitational torques (Section 3.1). We then establish the magnitude and size of gas motions driven in response to the spiral torquing (Section 3.2) and interpret the behavior in $\tau_{\text {dep }}$ in terms of gas kinematics in Section 4.1. We suggest that dynamical pressure plays a role in stabilizing GMCs.

Second, we develop a picture that links gas streaming motions with the gas depletion time using Bernoulli's principle, which equates an increase in gas velocity with a reduced external cloud pressure (Section 4.2). We use the radial profiles of streaming motions to assess the role of shear in the stability of the gas and we discuss the evidence against shock and stellar feedbackdriven turbulent support in Section 5.1. We conclude with a discussion of the broader implications of this result, from the angular offset between gas and young star tracers to the scatter in the KS SF relation (Section 5.2). In Section 5.3, we present a new form for the SF relation between $\Sigma_{\text {SFR }}$ and $\Sigma_{\text {gas }}$ that smoothly links SF at low $z$ and high $z$ by parameterizing the environmental dependence of clouds in terms of non-circular gas streaming motions. In particular, we predict a link between gas depletion time and gas fraction, as recently observed locally (Saintonge et al. 2012) and in evolution from $z=2$ (Combes et al. 2013).

\footnotetext{
10 In the extragalactic context, the gas depletion time is equated with the inverse of the SF efficiency $\mathrm{SFE}=\Sigma_{\mathrm{SFR}} / \Sigma_{\mathrm{gas}}$, although the former quantity is not a traditional efficiency, but an efficiency per free-fall time. This is in contrast with the SFE $\epsilon$ referred to in Galactic studies, which represents the fraction of gas converted into stars per SF event.
}

\section{DATA}

The analysis in this paper is based on $\mathrm{CO}(1-0)$ data obtained by PAWS (Schinnerer et al. 2013). PAWS observations cover a roughly $270^{\prime \prime} \times 170^{\prime \prime}$ field of view in the inner disk of M51 and include both interferometric data from the Plateau de Bure Interferometer (PdBI) and single-dish data from the IRAM $30 \mathrm{~m}$ telescope. The effective angular resolution in the final combined data cube is $1^{\prime \prime} .16 \times 0$.' 97 , or a spatial resolution of $\sim 40 \mathrm{pc}$ at our assumed distance of M51. The channel separation is $5 \mathrm{~km} \mathrm{~s}^{-1}$. The PAWS observing strategy, data reduction and combination procedures, and flux calibration are described by Pety et al. (2013).

Here, we use maps of the zeroth, first, and second moments of the PAWS data cube as presented by Pety et al. (2013). From the PAWS map of integrated $\mathrm{CO}(1-0)$ intensities (moment zero), we estimate the molecular gas surface density, applying a constant conversion factor $X_{\mathrm{CO}}=2 \times 10^{20} \mathrm{~cm}^{-2}\left(\mathrm{~K} \mathrm{~km} \mathrm{~s}^{-1}\right)^{-1}$ (as argued in Colombo et al. 2013a) and including a factor of 1.36 to account for the presence of helium. From the line-of-sight velocity field (first moment), we derive estimates for the circular velocity and the non-circular motions. The velocity dispersion map (second moment) supplies our measure of the turbulent gas motions after removal of the contribution from unresolved bulk motions estimated from the line-of-sight velocity field (adopting the formalism developed by Pety et al. 2013). We refer the reader to Pety et al. (2013) for a study of the resolution dependence of observables such as the maximum $\mathrm{CO}$ brightness (i.e., the gas surface density) and velocity second moment (gas velocity dispersion) measured from the PAWS data cube.

The PAWS data are complemented by an extensive multiwavelength data set, which has been assembled and described in Schinnerer et al. (2013). Here, we make use of the $S^{4} G$ IRAC $3.6 \mu \mathrm{m}$ image (Sheth et al. 2011), corrected for the contribution of non-stellar emission (Meidt et al. 2012), to define the stellar mass distribution. As described in Appendix B, we use this stellar mass map to derive the stellar potential and the profile of present day torques. We also use the $\mathrm{H} \alpha$ image (corrected for the stellar continuum using the procedure of Gutierrez et al. 2011) and MIPS $24 \mu \mathrm{m}$ data acquired as part of SINGS (Kennicutt et al. 2003) to trace the SF in M51. The MIPS $24 \mu \mathrm{m}$ image has been processed with the HiRes algorithm, giving a limiting angular resolution of 2".4 (Dumas et al. 2011). We combine the two images to trace the total (obscured and unobscured) SF following Leroy et al. (2009), using the empirical calibration of Calzetti et al. (2007).

\section{GAS FLOW AND GLOBAL STAR FORMATION PATTERNS IN M51}

M51 is a favorite test bed for spiral arm density wave theories. Studies of the spiral morphology and kinematics show evidence for the offset alignment of the gaseous, young, and old stellar tracers predicted by theory, as well as strong non-circular gas streaming motions (Vogel et al. 1993; Garcia-Burillo et al. 1993; Rand 1993; Aalto et al. 1999; Schuster et al. 2007; Shetty et al. 2007; Schinnerer et al. 2013). In this section, we examine gas motions in M51 using two independent approaches. We calculate the present-day torques on the molecular gas due to the stellar component. We then decompose the CO velocity field and show that the gas motions implied by our kinematic analysis are in excellent agreement with the response that we would expect based on our torque map. Finally, we compare our picture of gas flows with the global pattern of SF. 


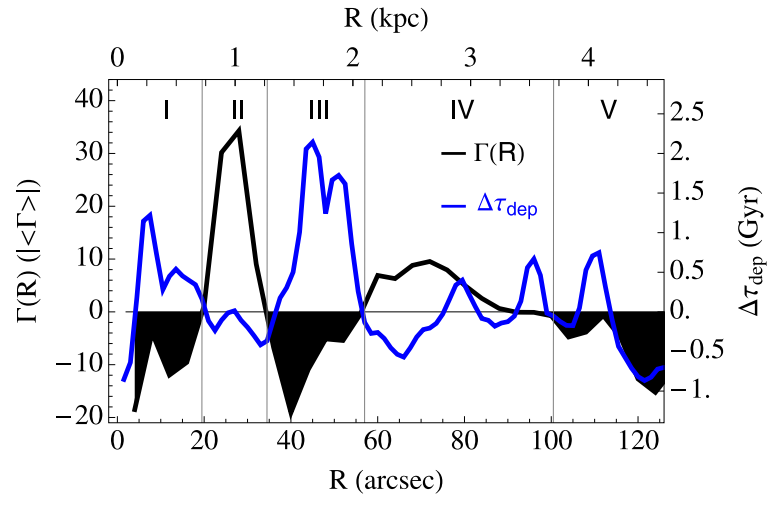

Figure 1. Radial profiles of the azimuthally averaged torque (in units of the absolute magnitude of the average torque across the PAWS field of view $|\langle\Gamma\rangle|$; black and white) and molecular gas depletion time $\Delta \tau_{\text {dep }}=\tau_{\text {dep }}-\left\langle\tau_{\text {dep }}\right\rangle$ (where $\left\langle\tau_{\mathrm{dep}}\right\rangle$ is the average $\tau_{\mathrm{dep}}$ across the PAWS field of view; blue) measured in 2".4 radial bins. Each crossing from negative to positive torque corresponds to the location of the $\mathrm{CR}$ of the structure: inside the $\mathrm{CR}$, material is driven radially inward and outside the CR, material moves outward.

\subsection{Present-day Torques}

As described in detail in Appendix B, our two-dimensional (2D) map of the old stellar light in M51 (Schinnerer et al. 2013) gives us a snap shot of the torques exerted by the nonaxisymmetric structure (i.e., nuclear bar, spiral arms) present in the density and hence gravitational potential of the system. These torques drive radially in- and outward gas motions, depending on the sign of torque. Crossings from negative to positive in the azimuthally averaged torque profile (Figure 1) mark the location of corotation, which is defined as the radius where the angular speeds of the disk and non-axisymmetric structure are the same. Inside (outside) corotation, negative (positive) torques drive gas radially inward (outward). Crossings from positive to negative torque coincide with the switch in predominance to a unique pattern.

This series of zero-crossings defines a set of dynamical environments (see Appendix B): the zone dominated by the nuclear bar $\left(R \lesssim 20^{\prime \prime}\right)$, the molecular ring $\left(R \sim 30^{\prime \prime}\right)$, the inner spiral $\left(30^{\prime \prime}<R<85^{\prime \prime}\right)$, and the outer spiral $\left(R>85^{\prime \prime}\right)$. The inner spiral can be further split into two zones, inside and outside the main spiral's corotation radius (CR) at $R=60^{\prime \prime}$. In what follows, we use this series of dynamical environments-and the torques within them-as the basis for interpreting the observed pattern of non-circular gas streaming motions in the inner disk of M51.

\subsection{Radial and Azimuthal Streaming Motions}

If our profile of the present-day torques in M51's inner disk is correct, then we expect the signatures of the gas motions that occur in response to the torques to be evident in the velocity field derived from the $\mathrm{CO}$ data. In Appendix $\mathrm{C}$, we use our mass-based model of the circular motion in M51 (Appendix A) to isolate the contribution of non-circular motion to the line-ofsight velocity field of the $\mathrm{CO}$ emission. Then, we decompose this non-circular motion into its radial and azimuthal components $v_{r}$ and $v_{\phi}$.

Figure 2 shows radial profiles of both reconstructed velocity components, which are calculated using $\mathrm{CO}$ intensity as a weighting factor in binned azimuthal averages to reveal the velocities characteristic of the (CO bright) spiral arms. Profiles with inverse-intensity weighting, which highlight the interarm streaming velocities, are qualitatively similar but show

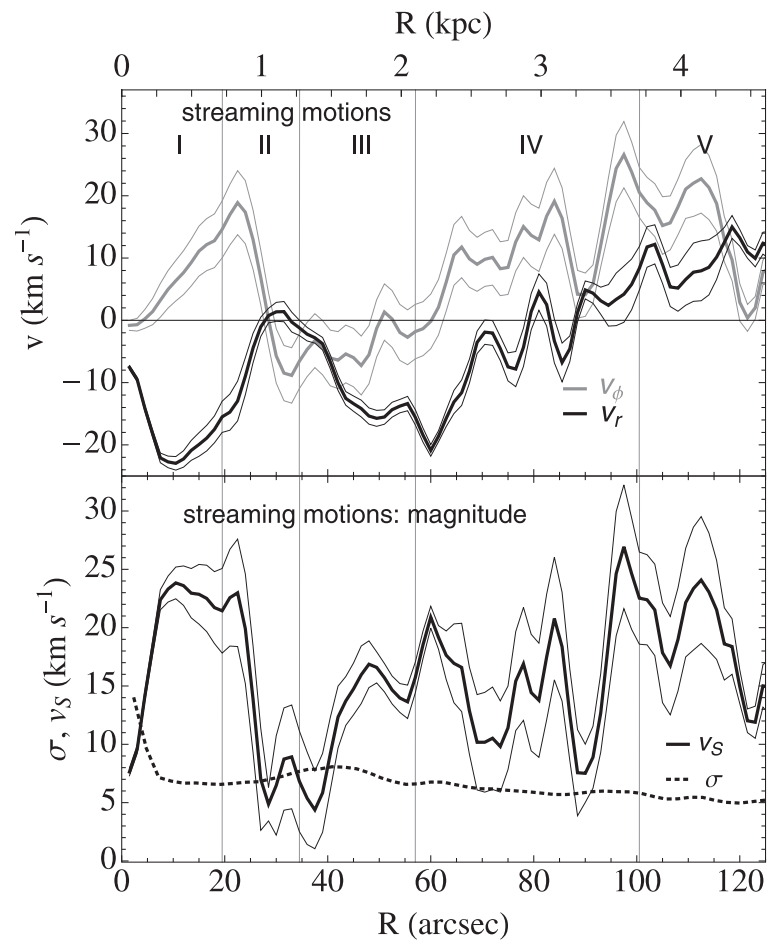

Figure 2. (Top) CO intensity-weighted radial (black) and azimuthal (gray) non-circular streaming velocities measured in $1^{\prime \prime} .5$ radial bins, reconstructed as described in the text. The intensity-weighting focuses our measurements on the CO-bright spiral arms. Thin lines represent the rms dispersion in solutions comprised of $\pm 5^{\circ}$ variations in the assumed spiral arm pitch angle $i_{p}$ added in quadrature with the uncertainty introduced by our adopted rotation curve (see the text). (Bottom) Magnitude of the non-circular streaming velocities $\left|v_{S}\right|=\sqrt{v_{r}^{2}+v_{\phi}^{2}}$ (black), derived from the non-circular motions above. Thin lines show the uncertainty measured from the errors above. The gas velocity dispersion profile $\sigma$-calculated as the azimuthal average of the line-of-sight dispersion corrected for unresolved bulk motions (i.e., Pety et al. 2013) - is shown for comparison (dotted line).

velocities $\approx 5-10 \mathrm{~km} \mathrm{~s}^{-1}$ lower than the profiles in Figure 2 (see Appendix C).

Our reconstructed radial velocities present us with a picture of radial gas flow that is qualitatively similar to that implied by the present-day torques. We once again retrieve the pattern of radial inflow and (weak) outflow that marks the locations of at least two distinct CRs, at nearly the same radii as found with the torque map (Figure 2). Radial velocities go to zero and switch sign near $25^{\prime \prime}$ and again between the range $60^{\prime \prime}<R<80^{\prime \prime}$, as predicted by the torques. Meanwhile, the azimuthal velocities exhibit similar zero-crossings as well as a pattern of positive and negative values that reflect a complex response to the spiral arm; depending on location relative to the spiral, gas at some points is stationary in azimuth or even flows opposite to the direction of rotation. There is some dependence on the adopted rotation curve (and hence stellar $M / L$ ), which shifts the azimuthal velocity horizontally in Figure 2. But, as described below, this choice does not greatly alter the measured magnitude of the streaming motions.

Almost independent of the particular rotation curve (or adopted stellar $M / L$; see Appendix C), the regions of negative torque identified in Figure 1 map to regions of the highest non-circular velocities in the arm. The magnitude of streaming motions $\left|v_{S}\right|=\sqrt{v_{r}^{2}+v_{\phi}^{2}}$ is shown in the bottom panel of Figure 2 for each of our three possible rotation curves, i.e., assuming the nominal $M / L=0.45 \pm 0.15$ (chosen as described 
in Appendix C). Although we do not expect negative torques to coincide with the largest streaming motions as a rule, the link between $v_{r}$ and $v_{S}$ is expected, according to the predicted relation between radial and azimuthal velocities in response to a spiral potential perturbation (i.e., Binney \& Tremaine 1987): for an $m$-armed spiral rotating at pattern speed $\Omega_{p}$ in a galaxy with a flat rotation curve (zero background shear) $v_{\phi}=v_{r} 2 \Omega / m\left(\Omega-\Omega_{p}\right)$ (with $\Omega$ the disk angular velocity) and so the total magnitude of streaming motions will follow $v_{r}$, even at the CR since $v_{r} \propto m\left(\Omega-\Omega_{p}\right)$.

\subsection{A Link between Gas Flows and Star Formation Efficiency in M51's Inner Disk}

A number of previous studies have shown that the pattern of M51's SF exhibits a strong radial dependence (see Schinnerer et al. 2013 and references therein). While SF falls on the convex side of the spiral very clearly between $60^{\prime \prime} \lesssim R \lesssim 80^{\prime \prime}$ ( $2 \mathrm{kpc} \lesssim R \lesssim 3 \mathrm{kpc}$ ), the young stars and gas coincide in the outer material arms. Meanwhile, there is little, if any, evidence of SF in the central 20" (in the nuclear bar zone; but see below) and between $35^{\prime \prime} \lesssim R \lesssim 60^{\prime \prime}$ in either $\mathrm{H} \alpha$ or $24 \mu \mathrm{m}$ emission. ${ }^{11}$ The radial dependence in SF activity in M51 causes the measured $\mathrm{SF}$ rate per unit of gas, or SF efficiency $(\mathrm{SFE})=\Sigma_{\mathrm{SFR}} / \Sigma_{\text {gas }}$ (i.e., Leroy et al. 2008), and hence gas depletion time $\tau_{\mathrm{dep}}=\mathrm{SFE}^{-1}$, to vary by as much as a factor of six throughout the inner $9 \mathrm{kpc}$. This same radial variation is evident in the SFE profile calculated by Leroy et al. (2009). Knapen et al. (1992) identified this same pattern and loosely interpreted this as a dependence of the SFE on location relative to spiral arm resonances.

In Figure 1, we compare the molecular gas depletion time $\tau_{\mathrm{dep}}$ to the profile of present-day gravitational torques. To calculate $\tau_{\text {dep }}$, we divide the radial profile of the molecular gas surface density $\Sigma_{\mathrm{H}_{2}}$ by the SFR surface density $\Sigma_{\mathrm{SFR}}$ profile. ${ }^{12}$ By using azimuthal averages of $\Sigma_{\mathrm{SFR}}$ and $\Sigma_{\mathrm{H}_{2}}$, we minimize the unavoidable bias introduced by the spatial offset between gas and young stars that is predicted by density wave theory and well-known to appear along the spiral arms in M51.

It is clear from Figure 1 that there is a strong link-shown as an inverse correlation-between $\tau_{\text {dep }}$ and the gravitational torques, underlining a strong dependence of the SF on dynamical environment. Radially inflowing gas appears to be less efficiently forming stars. More generally, gas sitting near the CR forms stars more efficiently than gas that is away from the CR and in motion relative to the background potential. We expect this to apply in other systems where radial outflow motions could potentially coincide with low SFE.

In contrast with expectations for a universal gas depletion time (i.e., Bigiel et al. 2008; Leroy et al. 2009), it appears that the $\tau_{\text {dep }}$ in M51 shows a genuine and non-monotonic radial dependence. This was previously demonstrated by Schuster et al. (2007) out to $12 \mathrm{kpc}$, although with a slightly different radial trend and using a different SF tracer. In particular, they find a depression in the ratio $\Sigma_{\mathrm{H}_{2}} / \Sigma_{\mathrm{SFR}}$ in the zone of the nuclear bar. However, several factors complicate the reliable estimation of $\Sigma_{\mathrm{SFR}}$ and thus $\tau_{\mathrm{dep}}$ in this area in particular. The diffuse farinfrared contribution from dust heating by an older population

\footnotetext{
11 Both SF tracers show a strong deficit in the zone $35^{\prime \prime} \lesssim R \lesssim 60^{\prime \prime}$, in particular, ruling out the extinction of optical photons as the source of the radial variation in the inferred SFR.

12 The gas depletion time defined in this way is the time to deplete the molecular material, rather than the total gas, although the two timescales should be similar; the atomic gas surface density is a factor of 10 lower than the molecular gas surface density across the PAWS field of view (Schuster et al. 2007; Leroy et al. 2009).
}

of bulge stars, for one, may lead to an overestimation of the SFR. Age and/or metallicity gradients in the underlying old stellar population also lead to uncertainties in the stellar continuum subtraction of narrow band $\mathrm{H} \alpha$ imaging.

In this work, we do not find the same rise in $\Sigma_{\mathrm{SFR}}$ toward the center as Schuster et al. (2007) did, possibly because any diffuse component present in the $24 \mu \mathrm{m}$ image we use is surreptitiously minimized in combination with the continuum-subtracted $\mathrm{H} \alpha$, which shows a central deficit (Schinnerer et al. 2013). In light of these uncertainties in $\Sigma_{\mathrm{SFR}}$, we consider our estimates for $\Sigma_{\mathrm{SFR}}$ and $\tau_{\text {dep }}$ to be least reliable in the center. We note, however, that an increase in $\tau_{\text {dep }}$ toward the center, such as we find, might be expected given the high shear characteristic of these radii (see Figure 6).

Throughout the remainder of the paper, we focus primarily on the spiral arms, which show persistent variation in $\tau_{\text {dep }}$ that is more arguably real. The gap in SF between $35^{\prime \prime} \lesssim R \lesssim 60^{\prime \prime}$ is evident in multiple tracers, in contrast with the high $\Sigma_{\text {gas }}$ in this zone. This contrast is particularly obvious at the high resolution of the PAWS observations (Schinnerer et al. 2013). But even at lower resolution, it is quantitatively clear that the SFR is lower than expected for the relatively high gas column; fewer stars are formed than expected for a global SF law or universal $\tau_{\text {dep }}$. Furthermore, the pattern in $\tau_{\text {dep }}$ cannot be attributed to variation in $X_{\mathrm{CO}}$. There is little to no indication that $X_{\mathrm{CO}}$ varies across the PAWS field of view (indeed, M51 has a negligible metallicity gradient, as recently discussed in detail by Colombo et al. 2013a). Instead, we propose that the radial dependence in $\tau_{\text {dep }}$ reflects the influence of dynamics on cloud properties (see Section 4.2), following the large-scale organization of the ISM by the non-axisymmetric structure present in the disk.

\section{GMC EVOLUTION IN M51: THE IMPORTANCE OF DYNAMICAL PRESSURE ON CLOUD STABILITY AND STAR FORMATION}

\subsection{Dynamical Pressure: Qualitative Remarks}

In the previous section, we showed that there is a strong link between zones of strong gas flow (which, in M51, correspond to regions of radial inflow and negative torque) and regions where molecular gas appears to be inefficient at forming stars. This is especially striking in the inner spiral arms spanning $35^{\prime \prime} \lesssim R \lesssim 60^{\prime \prime}$, where the molecular gas reaches relatively high surface densities but is effectively devoid of high-mass SF activity, in stark contrast with models that propose that the SFR and gas are well correlated.

In particular, Figure 2 shows that the molecular gas exhibits a complex response to M51's underlying non-axisymmetric potential. The rates at which gas flows along and across the arms depend on position along the arm and these rates vary in magnitude relative to one another depending on distance from the CR. The length of time spent in the potential well of the arm depends on the perpendicular component of the velocity $v_{\perp}$, which is well approximated by the radial streaming velocity $v_{r}$ given the tightness of the spiral. How far gas travels along the arm before exiting on the downstream side depends on the azimuthal streaming velocity $v_{\phi}$. The smaller this rate, the more quickly gas moves into and out of the spiral, leaving little time for clouds to acquire mass (i.e., through collisions/agglomeration in the spiral arm), become gravitational unstable, and form stars. At the CR, both velocities are zero and the gas is stationary in the rotating frame. 
We can begin to appreciate the effect of such motion on clouds by considering the ratio $v_{S}^{2} / \sigma^{2}$, which is a ratio of timescales: the velocity dispersion $\sigma$ sets the "equalization" time, or the timescale for clouds to reach pressure equilibrium with their surroundings, and the streaming velocity $v_{S}$ sets a travel, or relocation, time. In this case, $v_{S}^{2} / 4 \sigma^{2}$ is a measure of the square of the ratio between the time it takes to pressure equalize and the time to cross two cloud lengths. ${ }^{13}$ With the introduction of non-circular motions $v \gtrsim \sigma$, clouds equalize less quickly simply because they do not remain in any one environment for long enough.

This fact is underlined in the bottom panel of Figure 13, which shows that the ratio of equalization to relocation timescales is large everywhere except near the CR where, by definition, the gas is motionless in the rotating frame. We therefore interpret the close (if qualitative) relation between $\tau_{\text {dep }}$ and $v_{S}^{2} / \sigma^{2}$ to suggest that moving clouds have insufficient time to collapse and form many stars. This can be viewed as an effective stabilization, quantifiable in terms of the dynamical pressure $P_{\mathrm{dyn}}=\rho v_{S}^{2}$. As described in the next section, dynamical pressure reduces the internal pressure of the streaming medium in which clouds are embedded. This reduces the surface pressure on clouds, countering the gravity of the cloud.

According to the timescale arguments above, the dynamical pressure does not "dissipate" in a cloud-crossing time, implying that dynamical pressure cannot be ignored. Of course, whether or not clouds are sensitive to changes in surface pressure depends on whether clouds are dynamically decoupled from their surroundings. This depends on the internal pressure of the cloud in relation to the external pressure. Observations of clouds at high-density contrast with the surrounding medium are usually taken to imply an extreme pressure imbalance and thus little sensitivity to environment (e.g., Krumholz et al. 2012 and references therein). However, Hughes et al. (2013a) have recently shown that the internal and external pressures of clouds in M33, the LMC, and M51 (among others) track each other, even if they may not be equal, similar to the clouds in M64 studied by Rosolowsky \& Blitz (2005).

We thus have reason to expect that pressure plays an especially important role in M51, not least because the high molecular-toatomic gas ratio signifies a very high midplane pressure (e.g., Blitz \& Rosolowsky 2004). This was discussed by Hitschfeld et al. (2009), who found that the hydrostatic pressure is tightly correlated with the fraction of molecular to atomic material, indicating that this physical parameter determines the formation of molecular gas from the atomic phase.

As shown in Figure 3, the surface density in the ambient ISM is comparable to, and in some places exceeds, the surface density of individual GMCs. This implies that the internal and external cloud pressures are similar and so we expect that clouds embedded in this medium will be to some degree pressure supported (see, e.g., Krumholz et al. 2009). A large fraction of clouds from across the PAWS field do, in fact, appear to be supported by pressure according to their measured virial parameters $\alpha \gtrsim 1$ (Colombo et al. 2013a). Clouds from the inner $3 \mathrm{kpc}$ where the surface densities in the arm lie well above $\Sigma_{\mathrm{H}_{2}}=100 M_{\odot} \mathrm{pc}^{-2}$ preferentially exhibit even higher $\alpha \gtrsim 2$ than clouds from elsewhere in the disk. These clouds, which are comparable to clouds under starburst conditions, will therefore be keenly aware of changes in surface pressure.

\footnotetext{
13 We adopt the two-cloud length crossing time for this particular ratio for consistency with the formalism in Section 4.2.
}

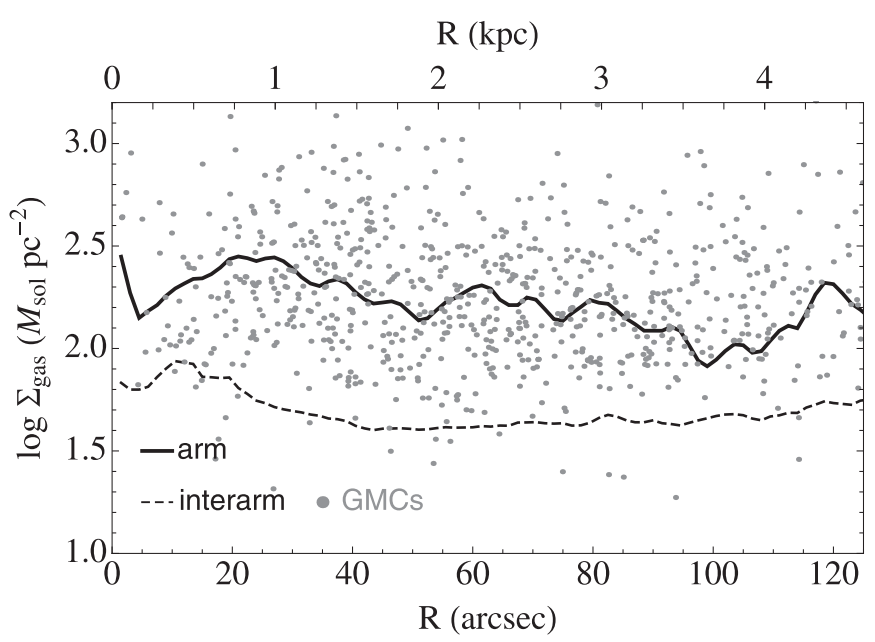

Figure 3. Molecular gas surface densities throughout the PAWS field. Cataloged GMCs (see Colombo et al. 2013a) are shown as gray dots, together with the average surface density in the arm region defined as in Colombo et al. (2013a) (black thick curve) and the average interarm surface density, calculated via inverse-intensity weighting (dashed thin curve).

Even in lower surface density environments, changes in surface pressure will affect cloud equilibrium, making the difference between stability and collapse. This may be the case in other spiral galaxies with lower molecular gas fractions than M51 since, even in these galaxies, arm surface densities will often lie near $\Sigma_{\mathrm{GMC}}$, even if the average surface density does not, preventing clouds from completely decoupling from their surroundings. For example, if we write the surface density of a gas disk hosting a two-armed spiral as

$$
\Sigma(R, \phi)=\Sigma_{0}(R) \cos (\phi)^{2 p}
$$

where higher (even integer) $2 p$ describes a thinner spiral, then the average gas surface density

$$
\langle\Sigma(R)\rangle=\frac{1}{2 \pi} \int_{0}^{\infty} \Sigma(R, \phi) d \phi=\Sigma_{0}(R) \frac{(2 p) !}{4^{p} p ! p !}
$$

will be $\sim 0.25-0.5$ times the peak surface density in the arm. According to the surface densities profiled by Leroy et al. (2008), we expect that, within the molecule-dominated zones in spiral galaxies, the gaseous spiral arms will supply non-negligible pressure support for GMCs.

\subsection{Dynamical Pressure: A Model Based on Bernoulli's Principle}

In the previous section, we established a qualitative correlation between the gas depletion time and dynamical pressure, which suggests that changes in the cloud surface pressure induced by gas kinematics influence cloud stability and therefore the ability of a cloud to form stars. To describe how these changes emerge, we next develop a simple model based on Bernoulli's principle, which equates an increase in gas velocity with a reduced pressure.

Specifically, we work under the assumption of isentropic flow of a compressible isothermal ideal gas and relate gas properties along the streamline between two zones using the compressible Bernoulli equation. This is a simplification and ignores the fact that gas in reality undergoes shocks and other forms of energy loss; gas in perturbed disks moving at non-zero velocities relative to the background rotating frame will exhibit transonic 
speeds (Mach number $M>1$ ). The shock can be weakened by strong gas self-gravity (e.g., Kim et al. 2008; Wada 2008) and we expect this to be true in M51. But in the frame of the shock, entropy is still not conserved and the values for the quantities assumed in the expressions in the following section will depart from the values assumed (the less so the weaker the shock).

In particular, under the assumption that the dominant source of pressure is turbulent in nature so that $P=\rho \sigma_{\text {turb }}^{2}$, we assume that the internal turbulent motion of the gas is uniform throughout the disk and take the average velocity dispersion $\sigma_{\text {turb }} \approx 10 \mathrm{~km} \mathrm{~s}^{-1}$ observed in M51 (Colombo et al. 2013b) as representative of this motion. This value takes into account the contribution of unresolved bulk motions to the observed linewidth, which we subtract (following the formalism adopted by Pety et al. 2013). Removing these motions yields a much more uniform velocity dispersion, although there is still a factor of two difference in the turbulent motions between the arm and interarm, likely because of the shock. Since this factor of two contrast is smaller than the density contrast between the arm and interarm (typically 5-10), we ignore it. We can more closely approximate reality by letting the density depend on pressure, i.e., by assuming compressibility.

Only with a better observational handle on energy losses and shocks in gas (which is minimal at present) will it be possible to invoke an expression equivalent to Bernoulli's but with the change in entropy included. This is something for which numerical calculation is better equipped and so we proceed with our simple calculation below with the aim of providing at least a coarse description of the phenomenon.

\subsubsection{The Stable GMC Mass Threshold}

In this section, we compare the maximum cloud mass stable against collapse in two different flows, i.e., in the arm and interarm regions. Gas moving at high relative velocity, tunneled along and through the arms, will result in a reduced external surface pressure for clouds forming in the arm. This then increases the maximum mass stable against collapse, as related by expression for the Bonnor-Ebert mass $M_{\mathrm{BE}} \propto$ $P^{-1 / 2}$ calculated from pressure, rather than virial, equilibrium, i.e., including a (thermal) surface pressure term. ${ }^{14,15}$ As a result of the dynamical pressure, (1) any collapse-unstable clouds that form will be of higher mass than virialized clouds and (2) fewer clouds will be collapse-unstable, leading to a lower SFE.

We can estimate the fractional increase in the Bonnor-Ebert mass as a result of dynamical pressure by equating the energy densities of gas in two zones with and without strong streaming motions (i.e., in the arm and interarm) in the non-inertial (rotating) reference frame. For simplicity, we use the compressible Bernoulli equation to relate gas properties along the streamline between the two zones. For pressure $P$ dominated by turbulent motion with dispersion $\sigma$ (i.e., $P_{i}=\rho_{i} \sigma^{2}$; note that this ignores an enhancement in cloud internal turbulence during passage through the arm), the pressure ratio of gas in zone 1 and zone 2 can be expressed as

$$
\ln \frac{P_{1}}{P_{2}}=\frac{v_{2}^{2}}{2 \sigma^{2}}-\frac{v_{1}^{2}}{2 \sigma^{2}}+\frac{\sqrt{2 \pi} G}{\sigma^{2}}\left(\Sigma_{2} h_{2}-\Sigma_{1} h_{1}\right),
$$

\footnotetext{
14 Note that the Bonnor-Ebert mass is equivalent to the Jeans mass ignoring any external or dynamical pressure.

15 Even if the surface term was of kinetic origin (rather than thermal, as assumed in the expression for the Bonnor-Ebert mass), we would expect an analogous change in the stable mass with a change in surface pressure, such as considered by Ballesteros-Paredes et al. (1999).
}

where $h_{i}$ is the gas scale height and $\Sigma_{i}$ is the gas surface density in the two zones. The first two terms on the right are the dynamical pressures, while the third represents the change in the hydrostatic pressure from zone to zone. Omitted terms include the stellar density factor in the hydrostatic pressure and other energy losses/gains.

As we are near enough to hydrostatic equilibrium so that $\Sigma_{1} / \Sigma_{2} \approx h_{2} / h_{1}$, the third term on the right is negligible and the first two terms dominate.

Since the fractional increase in the stable (Bonnor-Ebert) cloud mass is

$$
\frac{d M}{M_{1}}=-\frac{d P}{2 P_{1}}
$$

then

$$
\ln \frac{M_{2}}{M_{1}} \approx \frac{v_{2}^{2}-v_{1}^{2}}{4 \sigma^{2}} .
$$

From this point, we can see that streaming motions raise the cloud stable mass above the virial mass by

$$
\log \frac{M}{M_{\mathrm{vir}}} \approx \frac{v_{S}^{2}}{4 \sigma^{2}} \frac{1}{\ln 10},
$$

where the non-circular streaming in zone 2 is given by $v_{S}$ and zone 1 is identical to zone 2 except now its clouds are free of streaming (and dynamical pressure) and thus assumed to be virialized.

Within gas rotating in the plane of the galaxy, the stable mass is raised for all clouds undergoing non-circular motion. The stable mass for clouds moving at streaming velocity $v_{S} \sim 15-20 \mathrm{~km} \mathrm{~s}^{-1}$, typical of spiral arm streaming in M51, will be at least 1.5-2 times higher than for virialized clouds with similar surface densities and line widths $\sigma \lesssim 10 \mathrm{~km} \mathrm{~s}^{-1}$. As a result of the reduced surface pressure, collapse-unstable clouds that go on to form stars will be on average 1.5-2 times more massive than virialized clouds.

The difference from arm to interarm cloud masses should be similar, given the weaker streaming in the interarm. Conservatively allowing a factor of 1.5 difference in $v_{S}$, then we expect bound cloud masses to be on average 0.5 dex higher in the arm than in the interarm, very nearly what is measured from the cloud-decomposed PAWS CO(1-0) emission (see Colombo et al. 2013a; evident from the horizontal offset in the mass spectra for clouds in the two zones). Again, this ignores real variation in the gas dispersion from arm to interarm, but we note that the change in measured cloud line width between these two environments in M51 is only modest (Colombo et al. 2013a).

$$
\text { 4.2.2. Impact on } \tau_{\mathrm{dep}}
$$

In the previous section we showed that, as a consequence of strong non-circular streaming motions, the collapse-unstable clouds that go on to form stars will be on average 1.5-2 times more massive than virialized clouds. This suggests that starforming clouds in the arm (where streaming is high) preferentially host massive SF. But at the same time, any clouds in the arm below $\sim$ two times the virial mass will not collapse and form stars. This naturally translates into a decrease in the SFE, considering the change to the fraction of the total gas reservoir in the form of collapse-unstable clouds per free-fall time.

Specifically, assuming that the process (or processes) responsible for the build up of GMCs results in a mass spectrum

$$
N(>M) \propto\left(\frac{M}{M_{\max }}\right)^{\gamma+1},
$$



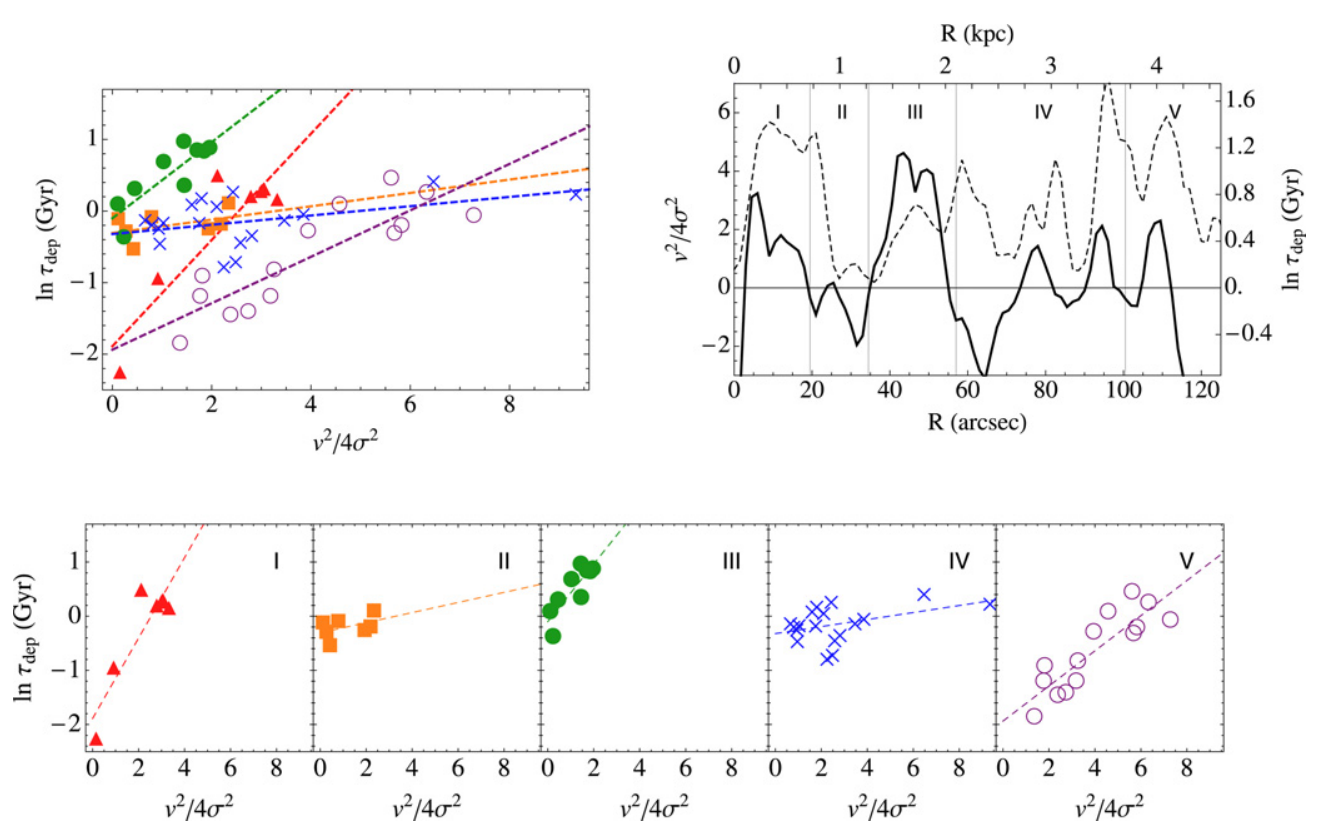

Figure 4. (Top left) Scatter plot of $\ln \tau_{\text {dep }}$ vs. $v_{S}^{2} / 4 \sigma^{2}$ extracted in 2 ". 4 bins from the curves at right. The symbols and color scale varies as at right, according to dynamical environment. Best-fit lines for each environment are overlaid. (Top right) Profiles of $\ln \tau_{\text {dep }}$ (solid black) and $v_{S}^{2} / 4 \sigma^{2}$ (dashed black) throughout M51. The measurements of $v_{S}$ and $\sigma$ used here are shown in the bottom panel of Figure 2. Errors have been omitted for clarity. Dynamical environments defined in Appendix B.2 (and described by Colombo et al. 2013a) are designated by color overlays: nuclear bar (red), molecular ring (orange), spiral inside CR (green), spiral outside CR (blue), and material pattern (purple). (Bottom) Scatter plots of $\ln \tau_{\text {dep }}$ vs. $v_{S}^{2} / 4 \sigma^{2}$ separated by environment.

then from the mass in (unstable) clouds above the stable threshold

$$
M\left(>M_{\text {stable }}\right)=\int_{M_{\text {stable }}}^{M_{\max }} \frac{d N(M)}{d M} M d M
$$

we see that the mass fraction of such clouds $F=$ $M\left(>M_{\text {stable }}\right) / M_{\text {total }}$ in a region of total mass $M_{\text {total }}$ will change as

$$
d F=(1+\gamma)\left(\frac{M}{M_{\max }}\right)^{\gamma} \frac{d M}{M_{\text {total }}}
$$

with a change to the stable mass by an amount $d M$.

Now, defining the average unstable cloud mass $\left\langle M_{c}\right\rangle=$ $M\left(>M_{\text {stable }}\right) / N$, then

$$
\frac{d F}{F}=(1+\gamma) \frac{d M}{\left\langle M_{c}\right\rangle} .
$$

We can cast this in terms of the SFE by recognizing that the collapse-unstable clouds-those above the stable thresholdare the clouds responsible for SF. Specifically, we write SFE = $F \beta$, where $\beta$ is the fraction of a cloud that is converted into stars per free-fall time (typically less than $5 \%$; e.g., Krumholz \& McKee 2005; Murray 2011). In this case, the fractional change to $\tau_{\mathrm{dep}}$, the inverse of the SFE, is given by $d \tau_{\mathrm{dep}} / \tau_{\mathrm{dep}}=-d F / F$, whereby

$$
\begin{aligned}
\ln \frac{\tau_{\mathrm{dep}}}{\tau_{\mathrm{dep}, 0}} & \approx-(1+\gamma) \ln \frac{M}{\left\langle M_{c}\right\rangle} \approx-(1+\gamma) \ln \frac{M}{M_{\mathrm{vir}}} \\
& =-(1+\gamma) \frac{v_{S}^{2}}{4 \sigma^{2}},
\end{aligned}
$$

using that $\left\langle M_{c}\right\rangle \approx M_{\mathrm{vir}}$ in the absence of streaming and where the constant of integration $\tau_{\mathrm{dep}, 0}$ is the unperturbed gas depletion time. By design, we take $\tau_{\text {dep }, 0}$ to be the depletion time in the case of virialized clouds.

\subsubsection{Comparing Model Predictions with Observations}

According to Equation (10), the gas depletion time, or inverse SFE, along the arm should proportionately follow the raised stable mass threshold in the presence of spiral streaming as measured by $v_{S}^{2} / 4 \sigma^{2}$. These two quantities are plotted as a function of radius in the left panel of Figure 4, using our measurements of the magnitude of streaming motions shown in the bottom panel of Figure 2 and the observed velocity dispersion $\sigma,{ }^{16}$ which is found to be relatively uniform across the disk, near $10 \mathrm{~km} \mathrm{~s}^{-1}$ (see Figure 2). With our very simple prediction, we are able to reproduce the observed pattern of SF; the radial variation of $\tau_{\text {dep }}$ nearly echoes the radial dependence in the kinematic term $v_{S}^{2} / \sigma^{2}$ to within a radially varying scale factor $-(\gamma+1)$.

The close match (albeit with scatter) is underlined on the right panel of Figure 4, where points fall along environmentunique lines with slopes $-(1+\gamma)$, according to Equation (10). Table 1 lists the slopes and intercepts of the best-fitting linear relationship in each of the five environments indicated in Figure 4 determined with the FITEXY routine (Press et al. 1992), assuming uncorrelated errors in both variables. We adopt 0.2 dex uncertainty in the measured $\tau_{\text {dep }}$, following Leroy et al. (2008), and the errors plotted in Figure 2 for the uncertainty in $v_{S}^{2} / 4 \sigma^{2}$. Despite the presence of scatter, the fitted relations are robust to changes in the radial range over which each dynamical environment extends. Increasing or decreasing the size of each by $10 \%$ leads to typically less than $10 \%$ variation in the fitted slope and intercept.

The average index $\gamma=-1.32 \pm 0.26$ measured from the best-fit slopes is consistent with (if slightly lower than) the indices measured in the same set of environments by Hughes

16 That is, we assume $\sigma$ describes the overall state of the gas (i.e., the combined thermal and kinetic pressure on the gas), including the contribution from turbulent motions. Note that independent of streaming, gas with higher $\sigma$ is expected to contain clouds with higher virial masses. 
Table 1

Environment-dependent Correlations

\begin{tabular}{|c|c|c|c|}
\hline Environment & $\gamma$ & $\tau_{\mathrm{dep}, 0}$ & $\chi^{2}$ \\
\hline I & $-1.75 \pm 0.16$ & $0.15 \pm 0.06$ & 5.30 \\
\hline II & $-1.1 \pm 0.18$ & $0.73 \pm 0.21$ & 0.88 \\
\hline III & $-1.54 \pm 0.24$ & $0.89 \pm 0.28$ & 1.98 \\
\hline IV & $-1.07 \pm 0.05$ & $0.72 \pm 0.13$ & 6.85 \\
\hline V & $-1.33 \pm 0.08$ & $0.14 \pm 0.04$ & 7.43 \\
\hline Environment & $\gamma$ & $\tau_{\mathrm{dep}, 0}$ & $\chi^{2}$ \\
\hline I & $-1.82 \pm 0.33$ & $0.52 \pm 0.12$ & 21.81 \\
\hline II & $-1.08 \pm 0.19$ & $0.79 \pm 0.15$ & 0.96 \\
\hline III & $-1.48 \pm 0.22$ & $1.54 \pm 0.25$ & 2.16 \\
\hline IV & $-1.07 \pm 0.05$ & $0.75 \pm 0.11$ & 6.6 \\
\hline V & $-1.15 \pm 0.04$ & $0.43 \pm 0.06$ & 13.35 \\
\hline Environment & $\gamma$ & $\tau_{\mathrm{dep}, 0}$ & $\chi^{2}$ \\
\hline I & $\ldots$ & $0.11 \pm 0.02$ & 9.25 \\
\hline II & $\cdots$ & $0.52 \pm 0.06$ & 26.74 \\
\hline III & $\cdots$ & $0.68 \pm 0.11$ & 14.03 \\
\hline IV & $\cdots$ & $0.14 \pm 0.02$ & 112.75 \\
\hline V & $\cdots$ & $0.04 \pm 0.01$ & 108.7 \\
\hline
\end{tabular}

Notes. Estimates of the cloud mass spectrum index $\gamma$ and fiducial gas depletion time $\tau_{\text {dep, } 0}$ measured from the slope and intercept, respectively, of the bestfitting straight line in each of the five distinct dynamical environments indicated in Figure 4 . The $\chi^{2}$ value of the fit is given in the right-most column. The five environments are, in ascending order, nuclear bar, molecular ring, spiral inside $\mathrm{CR}$, spiral outside $\mathrm{CR}$, and outer material pattern. The top third of the table lists the results of the fits shown in Figure 4. The middle third lists the results of fits to similar points, but that take into account interarm streaming, and the bottom third lists the $\tau_{\mathrm{dep}, 0}$ measured assuming a fixed slope corresponding to $\gamma=-1.72$, the average index measured via direct fitting of the cloud mass spectra in these same environments (Hughes et al. 2013a).

et al. (2013a) via direct fitting of the cloud mass spectra $\left(\left\langle\gamma_{\mathrm{df}}\right\rangle=\right.$ $-1.72 \pm 0.39$ on average). A $2^{\circ}$ error in the adopted inclination angle can easily explain this difference (see Section 4.2.4).

Moreover, the measured indices are remarkably compatible with the genuine, if modest, trend in the "direct-fit" $\gamma_{\mathrm{df}}$ with dynamical environment. In the study of Colombo et al. (2013a), variations in mass spectrum shape between the center, arm, and interarm environments is interpreted in terms of cloud formation, growth, and destruction mechanisms. We retrieve the record of these processes across the environments sampled here with our simple expression (but see Section 4.2.4).

Meanwhile, the best-fit intercepts of each environmentdependent relation correspond to a fiducial gas depletion time $\tau_{\text {dep }, 0}=0.5 \mathrm{Gyr}$, on average. This value represents the time to consume the molecular gas in the absence of spiral streaming and is notably shorter than the average depletion time across the PAWS field of view, $\left\langle\tau_{\text {dep }}\right\rangle=1.5$ Gyr. The latter value is consistent with molecular gas depletion times measured in the THINGS sample of nearby galaxies by Bigiel et al. (2008), Leroy et al. (2008), and Leroy et al. (2012). In some environments, the fiducial $\tau_{\text {dep }, 0}$ appears to be even shorter than 0.5 Gyr (see Figure 5; but see Section 4.2.4). Later in Section 5, we develop a picture that attributes departures from a fiducial, universal gas depletion time to motions in non-axisymmetric potentials.

We emphasize that the trend traced out in the right panel of Figure 4 does not arise with radial gradients in the kinematic term $v_{S}^{2} / 4 \sigma^{2}$ or $\tau_{\text {dep }}$ across the PAWS field. Variation appears on smaller (arcsecond) scales and the agreement between predicted and observed $\tau_{\mathrm{dep}}$ is genuine. This may be surprising, given that

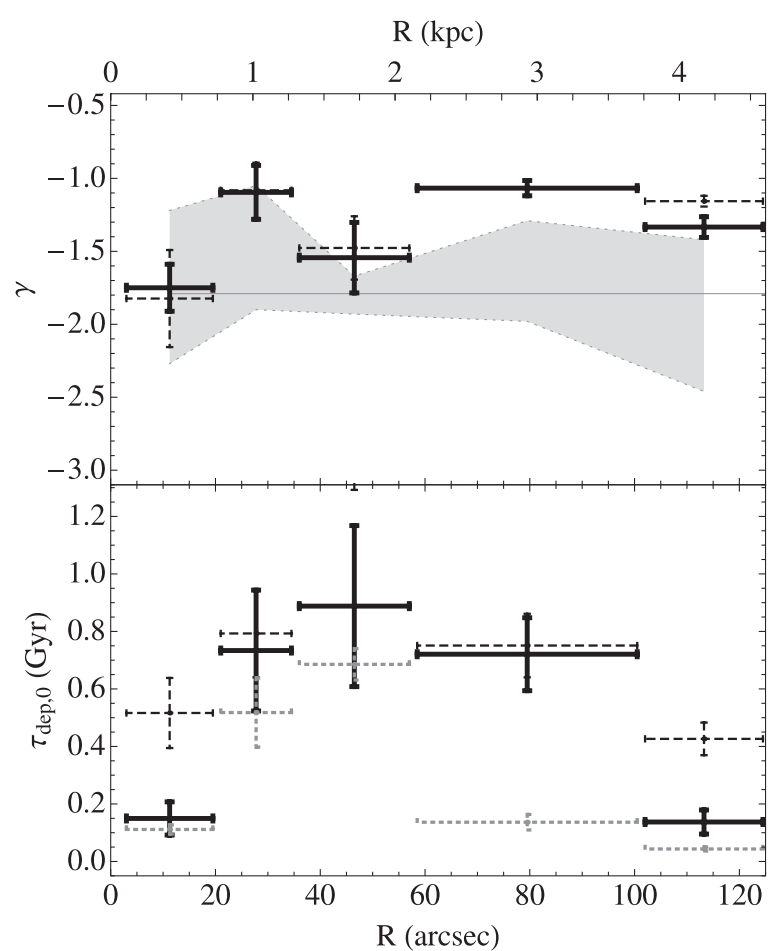

Figure 5. (Top) Cloud mass spectrum power-law index from within the series of M51 dynamical environments designated in Figure 4. The center of each environment is taken as the radial position of each measurement. Indices estimated from two independent approaches are shown: (1) the range spanned by the minimum and maximum indices measured from direct fits to the cloud mass spectra in each environment is shown in light gray (Colombo et al. 2013a; Hughes et al. 2013a). The gray horizontal line marks the average $\gamma=-1.72$. (2) The indices implied by the best-fit slopes in Figure 4 in each environment are shown in solid black with errors given by the statistical error in each measured slope. Best-fit slopes accounting for interarm streaming velocities are shown as thinner dashed lines. (Bottom) Fiducial gas depletion time $\tau_{\mathrm{dep}, 0}$ in the same series of M51 dynamical environments measured from the best-fit intercepts in the right panel of Figure 4 (solid black), the best-fit intercepts when interarm streaming velocities are included (thin dashed black), and the best-fitting $\tau_{\text {dep, } 0}$ with fixed $\gamma=-1.72$ (dotted gray).

our prediction is oversimplified, not least because it implicitly assumes a fixed, global, power-law mass spectrum. As revealed by Colombo et al. (2013a), cloud mass spectra in M51 show marked deviations from power-law behavior and uniformity along the arms coincident with changes in the dynamical environment defined here. These differences appear to indicate real variations in the mechanisms behind GMC formation, but they may also reveal how dynamical pressure plays a role in shaping/modifying an underlying global power-law mass spectrum (and a lognormal intensity probability density function; see Hughes et al. 2013b). Detailed modeling will be required to better understand and distinguish these scenarios.

\subsubsection{Sources of Uncertainty: Sensitivity to Observables}

Several factors influence the tightness of the correlation between $\tau_{\text {dep }}$ and $v_{S}^{2}$. In the previous section, we demonstrated that the overall scatter is dominated by real environmental variation in $\gamma$ and $\tau_{\text {dep }, 0}$. Within a given environment, the scatter that emerges tends to be on the order of 5\%-7\%, according to the errors on the measured slope and intercept of the best-fit linear relation. Both within and among environments, the correlation is subject to observational uncertainty, namely the accuracy in the SF and gas density tracers, the kinematic parameters assumed to deproject the line-of-sight velocities, and the pitch angle adopted in the calculation of the non-circular streaming motions. 
The reported statistical errors in the fitted $\tau_{\mathrm{dep}, 0}$ and $\gamma$ values primarily reflect uncertainty in the assumed pitch angle and rotation curve, which together define the errors in $v_{S}^{2}$ used as weights in the reduced $-\chi^{2}$ fit to the linear relation in Equation (10). In testing, we found that pitch angle variation is responsible for the largest uncertainty in the decomposition of the line-of-sight velocity field: by comparison, the radial and azimuthal streaming components are surprisingly robust to changes in the kinematic parameters, i.e., to the major axis position angle (P.A.) and inclination. We estimate that a P.A. variation of $\pm 5^{\circ}$ introduces a $10 \%$ uncertainty in the magnitude of the streaming motions and therefore up to a $20 \%$ change in the best-fit slope. Velocities extracted via solution in the spiral arm frame exhibit the expected $\sin i$ dependence. We expect inclination uncertainty, unlike pitch angle, to apply globally (under the assumption of an unwarped disk), resulting in an overall shift in the fitted $\tau_{\text {dep, } 0}$ and $\gamma$. A slightly higher inclination of $i=23^{\circ}$ would bring the average $\gamma$ into perfect agreement the average index measured by Hughes et al. (2013a).

To construct our estimate of the SFR, we use a combination of $\mathrm{H} \alpha$ and $24 \mu \mathrm{m}$ emission. This accounts for obscured and unobscured SF but does not explicitly include a correction for the diffuse component of the $24 \mu \mathrm{m}$ emission (e.g., as explored by Leroy et al. 2012), which is thought to arise with dust heating by an underlying older population of stars. The central bulge region (but also the spiral arms) most likely incorporates some level of dust emission that does not trace young stars, in light of the enhanced old stellar surface densities in this zone (i.e., as demonstrated in the bulge region of M31 by Groves et al. 2012). Underestimation of $\tau_{\text {dep }}$ in the center as a result is likely responsible for the particularly steep power-law index estimated for the nuclear bar environment, as well as the low $\tau_{\text {dep }, 0}$ there.

A low $\tau_{\text {dep }}$ in the center could alternatively arise from underestimation of the molecular gas surface density. Given the excellent sensitivity to low-level CO emission in the PAWS data set and little expected variation in $X_{\mathrm{CO}}$ (Hughes et al. 2013a; Colombo et al. 2013a), the latter would most likely occur only if the $\mathrm{CO}$ fails to trace a warmer molecular phase, e.g., as more regularly observed in the centers of galaxies (Dumas et al. 2010). The gas depletion time in the outermost material arm environment may also be artificially lowered due to the increased contribution from atomic gas component omitted at these radii (although this is still below 10\% at the edge of the PAWS field; see the profiles in Leroy et al. 2008).

Finally, the quality of the correlation depends on the reliability of our assumptions. We ignore any non-uniformity in the turbulent motions from arm to interarm as well as energy losses that occur in the spiral shock. Our isothermal assumption further ignores complex heating and cooling within the ISM. In reality, clouds will show variation in temperature between, e.g., the spiral arm and interarm regions. New simulations that implement heating and cooling prescriptions find dispersions in the range 3-7 $\mathrm{km} \mathrm{s}^{-1}$ (Hopkins et al. 2012; Dobbs et al. 2011). According to Equation (10), an error of this magnitude $(\sim 50 \%)$ would correspond to an uncertainty of $0.1 \mathrm{dex}$ in the gas depletion time ( $25 \%$ uncertainty in $\tau_{\text {dep }}$ ). As this is the typical measurement uncertainty in $\tau_{\text {dep }}$ (Leroy et al. 2008), our formalism can conversely accommodate $50 \%$ uncertainty in the velocity dispersion. Given the current data quality, we feel that the isothermal assumption is valid for our purpose, although future observations (i.e., with ALMA) will make it possible to test such a scenario.
Numerical calculations are clearly necessary to perform a detailed energy balance and follow the evolution and equilibrium of individual clouds during passage through the spiral arm. Still, the fact that we observe a correlation at all provides some indication that our simple expression is good to first order, even neglecting shocks and energy losses.

The form of the correlation we consider here (plotting $\ln \tau_{\text {dep }}$ versus $v_{S}^{2} / 4 \sigma^{2}$ ) by design represents the impact of dynamical pressure on the lengthening of the gas depletion time with respect to that of virialized clouds. But this ignores that, upon entering the arm, some clouds may themselves not be in virial equilibrium, i.e., due to non-zero streaming in the interarm. Even accounting for non-zero interarm streaming introduces little change to the correlation from environment to environment. We recalculated all fits including the interarm streaming velocities, i.e., according to Equation (4), where now the lengthening in gas depletion time in the arm is relative to the depletion time in the interarm. The $\gamma$ and $\tau_{\mathrm{dep}, 0}$ associated with the best-fit slopes and intercepts in each of the five environments under consideration are listed in the middle part of Table 1 . We find on average $\tau_{\text {dep }, 0}=0.66 \pm 0.26$ and $\gamma=-1.56 \pm 0.74$ and a trend with environment that similarly resembles that obtained from direct fits to the mass spectra, although with slight exaggeration (see the blue points in the top and bottom panels in Figure 5). Given that the interarm velocities are smaller than the arm velocities, this adjustment is responsible for less deviation from the simplest case than when we impose a global power-law mass spectrum. Estimates for $\tau_{\mathrm{dep}, 0}$ accounting for the interarm streaming are more similar to the values estimated including only arm streaming than those where the slope is fixed to $\gamma=-1.72$ (see the entries in the bottom part of Table 1 and plotted in Figure 5).

\section{DISCUSSION}

The close link between the predicted stable cloud mass and $\tau_{\text {dep }}$ described here strongly implicates the influence of dynamical pressure on the formation and evolution of the GMCs observed in M51. Indirect evidence for the role of surface pressure in cloud equilibrium is also seen in the lack of a clear size-linewidth relation for clouds in the arm (and interarm; Colombo et al. 2013a; Hughes et al. 2013a), which, when observed, is taken as evidence for virialized clouds. Our findings reiterate the warnings of Shetty et al. (2011) against the use of the virial parameter for determining cloud properties and quantifying boundedness. The external pressure-and the decrease in surface pressure in the presence of strong streaming motions in particular-must be taken into account.

The cloud-decomposed emission in the PAWS data cube shows other consistencies with the predictions for the impact of dynamical pressure. Colombo et al. (2013a) present evidence that the GMCs in the arms are more massive than those in the interarm by about an order of magnitude, clear based on the offset in the mass spectra for clouds in the two zones. Meanwhile, a larger fraction of the interarm gas is in the form of clouds than in the spiral arm (Colombo et al. 2013a). In our picture, regions with high streaming velocities should have fewer collapsing clouds and the few clouds that exist will be of higher mass than clouds that are virialized.

At present, the tight link between gas flows and SF is based on only one galaxy and tests of similar systems will be necessary to firmly establish the role of dynamical pressure. In this discussion section, we compare dynamical pressure with other sources of 


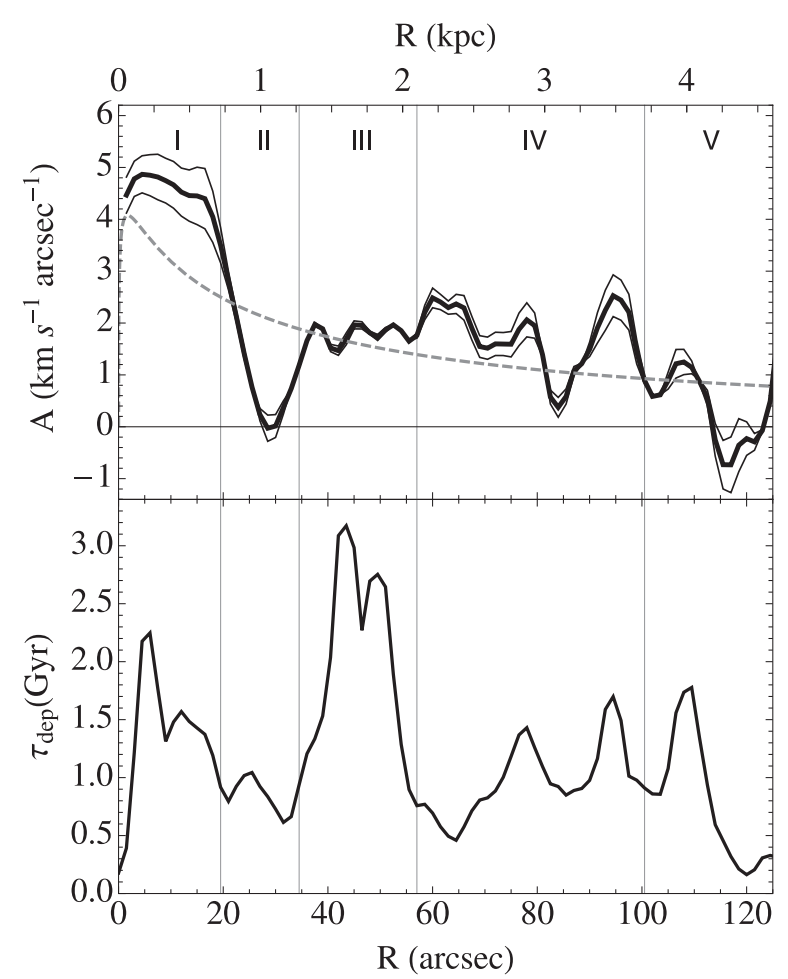

Figure 6. (Top) Profiles of the background shear due to differential rotation (gray dashed line) and the shear in the bar and spiral arm regions, calculated from our measure of $v_{\phi}$ in Figure 2 (black curve). Thin black lines represent the rms dispersion in solutions with $\pm 5^{\circ}$ variation in the assumed pitch angle $i_{p}$. (Bottom) For reference, the radial trend in the molecular gas depletion time is depicted in solid black, repeated from Figure 1.

cloud stability and highlight several aspects of SF that can be explained uniquely by our picture.

\subsection{Other Potential Sources of Cloud Stability}

In the previous section, we revealed an environmentdependent correlation between gas depletion time and dynamical pressure. This arises from the dependence of the SFE on the mass fraction of collapse-unstable clouds per free-fall time, which varies with location in the disk; the mass fraction of collapse-unstable clouds is specified by the environmentvarying cloud mass spectrum index, together with the stable mass threshold set by the dynamical pressure. In our picture, dynamical pressure effectively stabilizes the clouds and thus prevents all but those above the raised stable mass threshold from collapsing to form stars. But there are several other potential sources of cloud stability. We review these here and consider the possible role shocks might play in stimulating the observed pattern of SF. We conclude that none of these cases can adequately explain the observed pattern in the SFE or gas depletion time, leaving dynamical pressure as the most compelling source of stability.

\subsubsection{Shear}

As the locations of strong non-circular motions and enhanced surface density, spiral arms are preferentially sites of reduced shear (as argued by Elmegreen 1987, 1994; also see Kim \& Ostriker 2002, 2006). This also makes them favored sites of SF since, in the absence of shear to counter self-gravity, gas can form coherent cloud structures that become gravitationally unstable.
The link between enhanced shear and reduced SF has been studied by Seigar (2005; see also Weidner et al. 2010; Hocuk \& Spaans 2011). While this may hold on global scales, Figure 6 suggests otherwise for M51. The link between reduced shear and SF does not hold uniformly along the spiral arms on two counts: (1) shear as measured by the Oort parameter $A=$ $1 / 2\left(v_{\phi} / R-d v_{\phi} / d R\right)$ in the spiral arm is very clearly non-zero along the length of the arm. Shear in the spiral arm only counters the local background shear (from the rising rotation curve; see $\Omega$ in Figure 12) between $18^{\prime \prime} \lesssim R \lesssim 28^{\prime \prime}$ and further out near $R \sim 85^{\prime \prime}$ and $R \sim 100^{\prime \prime}$. In these outer regions, in particular, it appears that shear is responsible for the lengthening of the depletion time. (2) The region $35^{\prime \prime}<R<60^{\prime \prime}$ where we find a pronounced increase in $\tau_{\text {dep }}$ (or decrease in SFE) is characterized by a similar, if not lower, degree of shear as in the neighboring zone at larger galactocentric radius, which is characterized by relatively more SF. Moreover, as examined in Appendix D, the threshold for cloud instability and collapse in the presence of shear (as well as Coriolis and tidal forces) is exceeded by the observed gas surface density everywhere along the length of the arms. To prevent SF on cloud scales between $35^{\prime \prime}<R<60^{\prime \prime}$, another source of stability is clearly required.

Our finding echoes that of Dib et al. (2012), who considered the role of shear on the scale of individual molecular clouds in the Galactic Ring Survey. In all cases, they find that shear does not balance gas self-gravity and conclude that turbulent motions, stellar feedback, ${ }^{17}$ and/or magnetic fields are the principal agents of cloud stability.

\subsubsection{Turbulence and Magnetic Fields}

To produce the observed pattern in $\tau_{\text {dep }}$ in M51, we might similarly expect turbulent motions to be enhanced within clouds in the zone $35^{\prime \prime}<R<60^{\prime \prime}$ compared with clouds from neighboring zones. Our high-resolution PAWS observations, where the line-of-sight velocity profiles sample turbulent velocities above $\sim 2 \mathrm{~km} \mathrm{~s}^{-1}$ (Pety et al. 2013; Colombo et al. 2013a) on cloud scales, do not show such a pattern, however. The velocity dispersion shows no pronounced change along the arms (see Figure 2). The virial parameter $\alpha$, which measures the balance between the internal kinetic energy and gravity of clouds, shows no significant difference between this region of the spiral arm and the neighboring segment, beyond $R=60^{\prime \prime}$ (Colombo et al. 2013a). Either turbulent motions are not responsible for the radial variation in $\tau_{\text {dep }}$ shown in Figure 1 or they are present but fall below our velocity resolution. Below, we will consider shocks and their influence on cloud velocity dispersions in more detail. Here, we note that, given the marked absence of SF in this zone, cloud support cannot come from turbulence driven by stellar winds/feedback.

Magnetic fields are another potential influence on the organization of the ISM, but their role in cloud stabilization is not clear (or observationally established) at this point. Since they should pervade the ISM and clouds equally, it seems unlikely that they are responsible at the cloud level for the trends we see here.

\subsubsection{Shocks}

Spiral arm shocks, on the other hand, have been long appreciated for their role in the formation of GMCs and the triggering of

\footnotetext{
17 SF driven winds from successive SF events are thought to deposit the energy required to sustain turbulence (which otherwise dissipates quickly; i.e., Murray et al. 2010).
} 


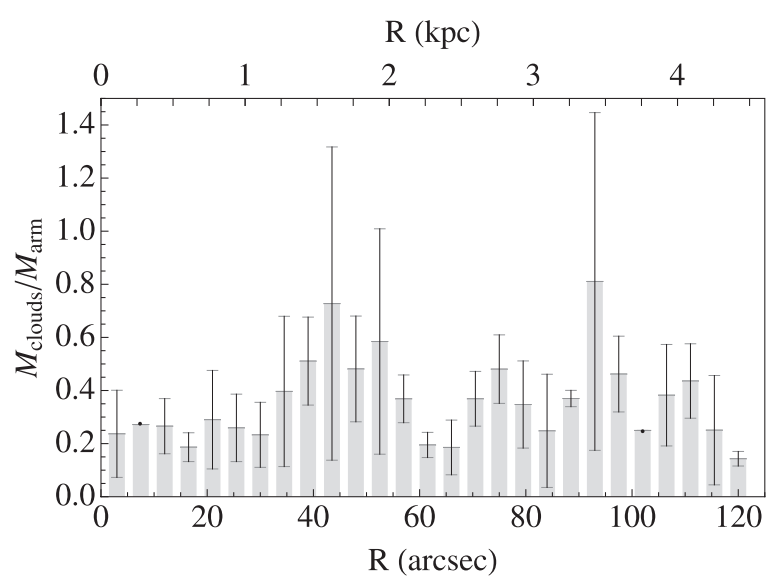

Figure 7. Histogram of the ratio of the molecular mass in cataloged clouds $M_{\text {clouds }}$ (estimated from the cloud CO luminosity; see Colombo et al. 2013a) to the total (luminous) mass in the arm $M_{\text {arm }}$ measured in $4 . .5$ radial bins. Thin lines delineate the dispersion in cloud masses within each bin.

SF (e.g., Roberts \& Stewart 1987; Gittins et al. 2003; Gittins \& Clarke 2004; Kim \& Ostriker 2002; Dobbs 2008). Shocks bring the gas surface densities high enough to initiate gravitational instability and at the same time favor massive GMC formation via collision and agglomeration. Clouds formed as a result of shocks are predicted to have elevated velocity dispersions (Bonnell et al. 2006), with clear implications for cloud boundedness and hence the global patterns of SF.

In this shock picture, variations in cloud properties would also presumably emerge along the spiral arms depending on the properties of the shock or as a result of variations in the balance between shear, self-gravity, and agglomeration (as well as the properties of the pre-shock medium; i.e., Dobbs \& Bonnell 2007; Dobbs 2008). For example, the zone we identified with very little SF might preferentially host unbound clouds formed via agglomeration in the shock rather than gravitational instability. However, there are several observations that counter this interpretation, as summarized below.

First, the surface density in this region is among the highest along the arms. In comparison with the other zones with $\mathrm{SF}$-and which presumably host bound clouds - this zone should likewise favor cloud formation via gravitational instability.

At these high surface densities, unbound clouds might still emerge if agglomeration builds on the masses of clouds already formed by the dominant process of instability in self-gravitating gas (as realized in the simulations of Dobbs 2008). The properties of these clouds are predicted to depend on the strength of the shock, with stronger shocks leading to the accumulation of more massive, unbound clouds (Dobbs \& Bonnell 2007; Dobbs 2008).

This case could potentially mimic a varying gas depletion time since, from this point, stars formed from individual bound clouds embedded in a larger unbound structure do so at an overall low efficiency. We might therefore expect the inverse of $\tau_{\text {dep }}$ to be well-matched to the ratio of total mass in clouds to the total mass in the arm (i.e., a low mass of potentially star-forming material where $\tau_{\text {dep }}$ is long). But Figure 7 shows no such behavior. Instead, the mass in clouds relative to the total mass seems even elevated where the SF is preferentially low. This is very strong evidence that the agglomeration of unbound cloud associations via shocks is not responsible for the observed pattern in $\tau_{\mathrm{dep}}$, especially since it conserva- tively assumes that all cataloged clouds will collapse to form stars.

As analyzed by Colombo et al. (2013a), the shape of the cloud mass spectrum provides a record of the processes responsible for the formation (and destruction) of clouds in M51. GMCs in the spiral arms are predominantly formed by gravitational instability, although agglomeration plays a role in organizing the ISM into cloud associations along these structures. Notably, the cloud mass spectrum shows no significant change in overall shape along the arm (Colombo et al. 2013a). This suggests that the mix of instability and agglomeration in cloud formation is equal in arm environments, namely between the spiral segment neighbored by SF on its convex side and the segment $30^{\prime \prime}<R<60^{\prime \prime}$ with little to no SF. The pattern in SF therefore does not appear to be tied to the cloud formation mechanism.

\subsubsection{Triggered Star Formation}

SF triggering by spiral-arm shock dissipation is also very clearly not responsible for the observed pattern in the SFE. Since stronger shocks are accompanied by larger velocity gradients and thus dissipate more energy, we might have expected relatively short gas depletion times in exactly the locations of strongest spiral shock (i.e., anywhere away from corotation), unlike what is observed. This picture would also require the shock strength to vary from one segment of the arm to the other (i.e., $40^{\prime \prime}-60^{\prime \prime}$ compared with $60^{\prime \prime}-80^{\prime \prime}$ ) to explain the difference in the SF properties between these two zones, but this does not appear to be the case (Schinnerer et al. 2013). Taking the spiral shock strength as the size of the offset between the molecular spiral arm from the potential minimum traced by the peak in the old stellar light distribution imaged at $3.6 \mu \mathrm{m}$, Schinnerer et al. (2013) find only a modest difference between the zones $40^{\prime \prime}-60^{\prime \prime}$ and $60^{\prime \prime}-80^{\prime \prime}$. We therefore conclude that, although spiral-arm shocks may be active in M51, it is not clear that they have a strong, direct influence on the triggering of SF.

\subsection{Implications for Patterns of Star Formation in Observed and Simulated Galactic Disks}

Up to this point, simulations and semi-analytical models of GMC formation and evolution rarely consider nonaxisymmetric potentials and so streaming motions and dynamical pressure have been neglected. Variations in the SFE and cloud stability have mostly focused on the role of turbulent support and turbulent energy dissipation (Krumholz \& McKee 2005; Murray et al. 2010; Feldmann et al. 2011). The few simulations with fixed spiral potentials have thus far focused only on very tightly wound spirals with pitch angles $i_{p}<10^{\circ}$ and so have not probed the regime of strong streaming motions. In addition, these simulations consider Bernouli's principle more for its role in spur formation via the Kelvin-Helmholz instability, which has subsequently been found to be ineffective in real galaxies compared with the magneto-Jeans instability (Kim \& Ostriker 2002).

From the observational perspective, we can already infer the role of dynamical pressure in galaxy disks. The strong dynamical dependence of the conversion of gas into stars presumably contributes to the frequent lack of a clear signature in the angular cross correlation between gas and young star tracers (Foyle et al. 2011; Egusa et al. 2009). The angular offset between the young stars and gas predicted as a result of the propagation of the spiral pattern (combined with the timescale for SF; e.g., Roberts \& Stewart 1987), will not be observed, 
even where the gas column is high, if the gas is dynamically prevented from forming stars.

The influence of dynamical pressure is also manifest at the global level. We suspect that it keeps stronger grand-design spirals from forming an excess of stars relative to weaker, flocculent spirals with similar global molecular gas surface densities, as observed (see Elmegreen \& Elmegreen 1986). Whereas stronger spirals result in locally higher gas surface densities and might therefore be expected to exhibit higher SFR surface densities, they also locally stabilize clouds through dynamical pressure. These two effects presumably together keep the global SFR independent of spiral strength. An increased velocity dispersion in the strongest spiral arms will achieve the same result, but we note that a distinction from dynamical pressure is difficult to establish, as observations at resolutions above the size scale of GMCs will also reflect unresolved streaming motions.

The strong internal gas flows associated with galaxy interactions suggests that dynamical pressure may be a common feature in such scenarios. M51 and its companion are an ideal test bed for exploring the impact of interactions on gas organization and subsequent SF. Our consideration of the impact of M51's companion galaxy has been so far indirect, concerning the non-axisymmetric structure across the PAWS field that the interaction helped to stimulate and shape. Features that are more directly influenced by the companion, namely the "bridge" that extends between the two galaxies and the opposite "tail," are beyond the edge of the PAWS field of view, precluding the study of the ISM organization and the properties and progression of SF with the same detail as we have undertaken at smaller galactocentric radius. As the ISM composition is changed at large radii as well, future insight into the nature of the impact of the interaction will require extended, high-resolution kinematic tracers of the molecular and atomic ISM phases.

\subsubsection{Relevance for Barred Galaxies}

The scenario we find here-low SFE in the CO-bright inner spiral arm segments_can be thought of as comparable to the dust lanes along the length of bars typically observed to harbor little SF (Sheth et al. 2005; Comeron et al. 2009). $\mathrm{SF}$ is thought to be inhibited in the strongest bars because of strong shocks and high shear (Athanassoula 1992; Sheth et al. 2005; but see Zurita et al. 2004, who suggest shocks help trigger SF). In our picture, on the other hand, the strong radially inward streaming motions that accompany strong bar torquing stabilize clouds, shutting off SF. We find this to be a compelling interpretation since the two conventional kinematic sources of cloud stabilization-enhanced shear and shockinduced turbulent motions - cannot be active in the comparable zone in M51 with high $\tau_{\text {dep }}$ (Section 5.1). Not only is shear reduced in this zone, but the gas is not characterized by extraordinary line widths, implying that the fewer collapsing clouds in this zone are not the result of preferentially enhanced turbulence.

Dynamical pressure can also be invoked, as an alternative to shear and shocks, to explain the suppression of SF in the Galactic center (Longmore et al. 2013). The observed gas kinematics in the Milky Way's central molecular zone are consistent with characteristic motions along bar orbits, but the evidence for inflow motions along a bar shock are less clear; the fall-off in the signature "parallelogram envelope" with increasing distance from the center in the $p v$ diagram (Binney et al. 1991) is not especially obvious. This could be due to the data quality (sensitivity issues), or it may indicate that the weak oval nuclear bar does not sustain shear and shocks. ${ }^{18}$ In this case, if shear is not present to stabilize clouds, dynamical pressure due to noncircular motions in the bar may be responsible for altering the ability of clouds to form stars. Estimates of the critical surface densities for shear and dynamical pressure (see Appendix D) could help further establish the likelihood of these two scenarios.

\subsection{Implications for Local and Global Star Formation Relations}

As demonstrated in Section 4.2.3, the influence of dynamical pressure manifests itself by introducing departures from a fiducial gas depletion time $\tau_{\mathrm{dep}, 0}$, with higher streaming motions leading to longer $\tau_{\mathrm{dep}}$. According to the formalism there, this fiducial $\tau_{\mathrm{dep}, 0}$ is representative of virialized clouds and our fitting in the case of M51 (Section 4.2.3) suggests that $\tau_{\mathrm{dep}, 0}$ can be as low as $\sim 0.2$ Gyr. By analogy with Krumholz et al. (2012), we view $\tau_{\mathrm{dep}, 0}$ as set by the cloud free-fall time $t_{\mathrm{ff}}$ modulo some roughly constant dimensionless efficiency $\epsilon_{\mathrm{ff}}(\sim 0.01$ in a supersonically turbulent medium; Krumholz \&McKee 2005) measuring the intrinsic SFE in a cloud. But this efficiency is modulated by the dynamical pressure term so that

$$
\tau_{\mathrm{dep}}=\frac{t_{\mathrm{ff}}}{\epsilon_{\mathrm{ff}}} e^{-(\gamma+1) v_{s}^{2} / 4 \sigma^{2}} .
$$

This change in the efficiency reflects the influence of the environment of a cloud on its SF properties. Alternatively, we can view the exponential term as altering the time required to complete collapse. This would be more similar to the Krumholz et al. (2012) picture, in which the free-fall time depends on the cloud "regime"; clouds that are dynamically coupled to their environment with a low density contrast have shorter depletion times than clouds that are decoupled, at high overdensity. The environmental dependence parameterized by this bimodal freefall time leads to a successful fit to observations ranging from star-forming clouds in the Milky Way to high- $z$ starbursts. Below, we show that our predictions can also explain the observations by presenting a form of the SF law that leads to an analogous environmental dependence. In contrast with the Krumholz et al. (2012) picture, our environmental dependence is smoothly parameterized by the dynamical pressure, which affects the coupling to the environment by reducing the cloud surface pressure.

\subsubsection{Impact on Local Trends}

With the change in the depletion time in Equation (11), the $\mathrm{SF}$ relation can be expressed as

$$
\Sigma_{\mathrm{SFR}}=\frac{\Sigma_{\mathrm{gas}}}{\tau_{\mathrm{dep}, 0}} e^{(\gamma+1) v_{s}^{2} / 4 \sigma^{2}} .
$$

We see immediately that the range in streaming velocities both within and among spiral galaxies should contribute to the scatter in the KS SF relation (i.e., Leroy et al. 2009; Bigiel et al. 2008). ${ }^{19}$ This is illustrated in Figure 8. The left panel shows measurements from annuli within M51, while these annuli are

\footnotetext{
18 Note that shear is expected to be zero in the absence of shocking due to gravitational or viscous torques on the gas within the zone of the bar.

19 Pixel-by-pixel comparisons between $\Sigma_{\text {gas }}$ and $\Sigma_{\text {SFR }}$ should reveal such departures from a universal gas depletion time most clearly; the $\tau_{\text {dep }}$ measured from larger-area averages is the true $\tau_{\text {dep }}$ weighted by $\Sigma_{\text {SFR }}$ and will thus less strongly track low SF environments.
} 

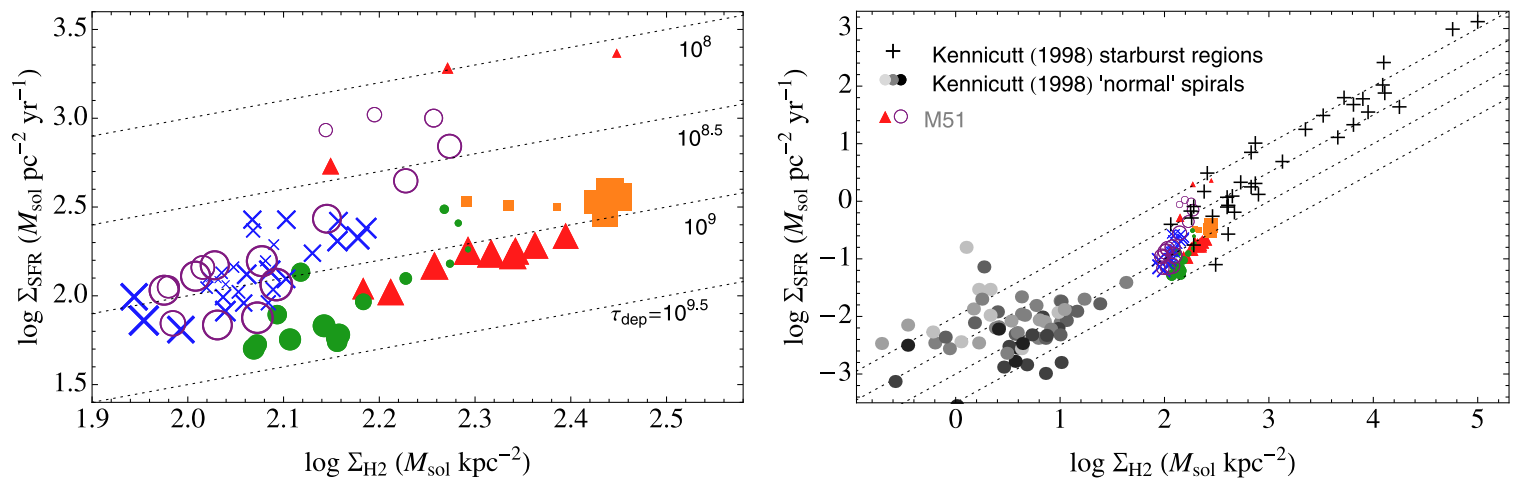

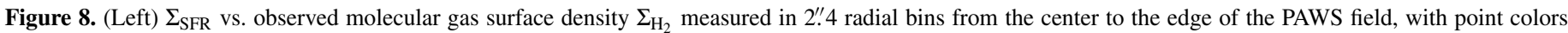

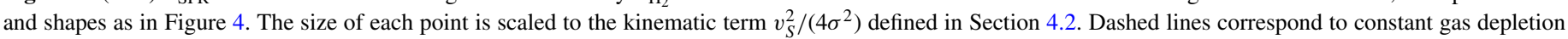

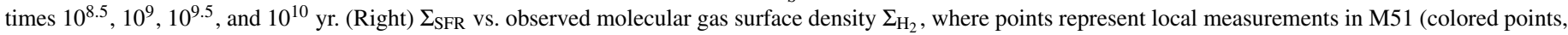

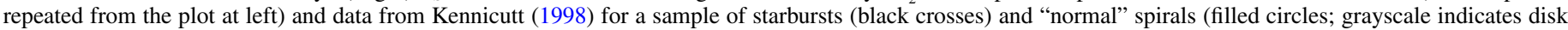
morphology, from (black) early-type to (light gray) late-type). Lines of constant depletion time are repeated from the plot on the left.

plotted together with integrated measurements from Kennicutt (1998) for normal spiral galaxies and starburst environments on the right.

Regions in M51 with the highest streaming motions sit at the longer gas depletion times, falling below the points with relatively less streaming. Spiral galaxies with similar non-circular motions will show a similar spread in (local) depletion time. But more importantly, given that typical maximum streaming velocities are $10-15 \mathrm{~km} \mathrm{~s}^{-1}$, we expect that spiral galaxies will preferentially show roughly the same maximum $\tau_{\text {dep }}$, especially since measurements on the scales of $1 \mathrm{kpc}$ and larger tend to be weighted to the bright, high-density spiral arms. Galaxies with little to no streaming, on the other hand, will show lower depletion times; the gas in these systems will form stars closer to the fiducial gas depletion.

In this light, we might view the gas depletion time $\tau_{\mathrm{dep}} \sim 2 \mathrm{Gyr}$ measured by Bigiel et al. (2008) for a sample of spiral galaxies (ranging in stellar mass from $\sim 10^{7}$ to $10^{11} M_{\odot}$ ) as characteristic of the depletion time in the presence of spiral streaming motions. At the same time, we can attribute the lower $\sim 1 \mathrm{Gyr}$ gas depletion times in the Kennicutt (1998) starbursts and also characteristic of low-mass systems assuming a Galactic conversion factor $X_{\mathrm{CO}}$ (Leroy et al. 2013; but see the discussion about the impact of $X_{\mathrm{CO}}$ below) to the fact that this SF is occurring in a relatively stable dynamical environment. In the former case, the SF typically occurs at resonances where the streaming motions are zero, while in the latter case, streaming is absent because these systems fail to develop the nonaxisymmetric structure that drives these motions (the low mass means the disk never becomes unstable).

\subsubsection{Impact on Global Trends}

In reality, we expect to see a range of gas depletion times intermediate between these cases. Since disks of a certain mass will be unstable to structure of a particular wavenumber and pitch angle $i_{p}$, as upheld in the density wave paradigm, we can express the $i_{p}$ dependence in terms of galaxy mass or circular velocity $V_{c}$ using the dispersion relation for $m$-armed density wave spirals (i.e., Binney \& Tremaine 1987). We use the perturbed continuity equation

$$
v_{r} \approx \frac{\left(\Omega-\Omega_{p}\right) \tan i_{p}}{m} \frac{\Sigma}{\Sigma_{0}}
$$

to express the angular velocity difference $\Omega-\Omega_{p}$ in terms of the radial streaming velocities $v_{r}$, where $\Omega_{p}$ is the wave/spiral pattern speed and $\Sigma\left(\Sigma_{0}\right)$ is the perturbed (unperturbed) gas surface density. In this way, we find

$$
\tan i_{p}=\frac{1}{4 \pi V_{c}^{2}}\left[v_{r}^{2}\left(\frac{\Sigma}{\Sigma_{0}}\right)^{2}-\sigma^{2}\right],
$$

where $V_{c}=\Omega R$ is the rotational velocity. This reproduces the well-known observation that spiral arm pitch angle increases with galaxy mass, $V_{c}$, or Hubble type (Kennicutt 1981).

Now from Equation (13), we predict that the magnitude of the streaming motions

$$
v_{S} \approx v_{r} \propto \frac{V_{c}}{m} \frac{\Sigma}{\Sigma_{0}}
$$

to lowest order in $V_{c}$. (Note that this applies globally, away from corotation, so that $v_{r} \sim v_{\phi}$ and $v_{S} \sim v_{r}$.)

Less tightly wound spirals in the more massive, early-type disks drive stronger streaming motions. This implies that spiral galaxies with higher mass (larger $V_{c}$ ) should exhibit longer gas depletion times. This is, in fact, what global measurements of $\Sigma_{\text {SFR }}$ and $\Sigma_{\text {gas }}$ suggest: the normal spiral sample in the KS plot on the right in Figure 8 indicates an ordering between depletion time and morphology/Hubble type. (Note that while the starbursts lie above M51 and the earlier-type spiral galaxies, they are more similar to the lower mass later-type galaxies, which exhibit similarly short depletion times.) This is even clearer in the HERACLES sample of galaxies, where integrated measures of the gas depletion time smoothly decline from early to late spiral types (Leroy et al. 2013).

This dependence of $v_{S}$ on $V_{c}$ accounts for the observed weak correlation between $\tau_{\text {dep }}$ and stellar mass among galaxies observed by Leroy et al. (2008, 2009) and Saintonge et al. (2012). According to Equation (13) or (15), the scatter in that relation at fixed stellar mass can be attributed to, e.g., morphologic variations (number and strength of arms, pitch angle) and gas content (see below). It can also explain the modest success of the "dynamical Schmidt" law in fitting observations, like other similar prescriptions that express the gas depletion time in terms of $V_{c}$ (i.e., Tan 2010 and references therein). Here, though, we predict a non-linear dependence $\tau_{\text {dep }} \propto e^{-V_{c}^{2} / \sigma^{2}}$ that can be approximated by $V_{c}$ only at low $V_{c}$ (and in particular at low $V_{c} / \sigma$ ). 


\subsubsection{High-z Star Formation Relation}

Our form of the SF relation is also capable of linking local and high- $z$ SF. Observations suggest that star-forming galaxies at earlier times form stars at a high efficiency compared with their local counterparts (i.e., Daddi et al. 2010), with depletion times comparable to those in local starburst environments. (Local and $z=2$ star forming disks fall on a non-linear KS relation with power-law index $n \sim 1.3$; e.g., Daddi et al. 2010; Krumholz et al. 2012.) High- $z$ starbursts and mergers are inferred to consume their gas even faster, giving the appearance of a bimodality in the KS relation at $z \sim 2$ (Daddi et al. 2010; Genzel et al. 2010). These authors suggest that these two modes of SF can be linked by adopting a form for the SF relation that incorporates the dynamical time, i.e., letting the dynamical time set the gas depletion time. However, Krumholz et al. (2012) demonstrate that this form of the SF relation cannot be consistently applied to local systems. Instead, the fiducial gas depletion time is argued to be lower in high- $z$ starbursts, likely due to the expected high densities that result under the compressive weight of the ISM. As is clearer below, our picture presents a parameterization of this weight in terms of $V_{c} / \sigma$ or the gas mass fraction $f_{g}$.

To more clearly illustrate the differences we expect to see between systems at low and high $z$, we alternatively express the streaming velocity in terms of the Toomre $Q$ and gas-to-total mass ratio $f_{g}$. Using

$$
Q=\frac{\sqrt{2(\beta+1)} \sigma \Omega}{\pi G \Sigma_{\mathrm{gas}}},
$$

where $\beta=d \ln \Omega / d \ln R=1$ for solid-body rotation and $\beta$ $=0$ in the case of flat rotation curves, $V_{c}^{2} \approx \pi G \Sigma_{\text {tot }} R_{\text {edge }}$, and assuming that the stellar and gaseous disks cover roughly the same area so that $f_{g}=\Sigma_{\text {gas }} / \Sigma_{\text {tot }}$ then, together with Equation (15), we find

$$
\frac{v_{S}}{\sigma} \propto \frac{\Sigma}{\Sigma_{0}} \frac{1}{m} \frac{\sqrt{2(\beta+1)}}{f_{g} Q} .
$$

Star-forming disks at high $z$ have lower gas depletion times than similarly marginally unstable disks at $z=0$ due to the higher gas fraction at earlier times. High- $z$ starbursts and merging systems can have even lower depletion times, given the likely higher gas fractions.

Note that the observed trends also follow from Equation (15), as the ratio $V_{c} / \sigma$ is lower in star-forming disks at earlier times and even lower in starbursting mergers (i.e., LIRGS/ ULIRGS), given the enhanced turbulence and larger $\sigma$. This may explain why a linear dependence of $\Sigma_{\mathrm{SFR}}$ on $V_{c}$ can be used to fit observations at high $z$, where the quantity $V_{c} /$ $\sigma$ is intrinsically low, and thus a suitable approximation of the exponential dependence we find (see Section 5.3.2). We emphasize, though, that $V_{c}$ is only part of the description, as the ratio $V_{c} / \sigma$ provides the more fundamental measure of the importance of dynamical pressure. As described in Section 4.1, even when streaming motions are present, dynamical pressure is not important if clouds can equalize before they undergo translation by more than a cloud length, that is, if $\sigma>v_{S}$. With Equation (15), this is equivalent to the condition $\Sigma_{\text {gas }} \sim \Sigma_{\text {tot }}$ for gas disks in hydrostatic equilibrium (recall that by writing Equation (15), we replace the existence of streaming motions by the requirement for a disk massive enough for instabilities to form). So even in the presence of streaming motions, a high gas fraction prevents an increase in the depletion time.
Consider, for example, the difference between starbursts at low and high $z$, which each have short depletion times but very different $V_{c}$. In the case of local starbursts, the high gas densities (and high SF) are possible because of the absence of motion relative to the background rotating potential; locally motionless gas (fed from neighboring regions) can retain and build a molecular reservoir. This scenario is different from the conditions that lead to bursts of SF at higher redshift, where accreted external gas builds up globally high surface densities and streaming motions themselves can be quite high (i.e., given the high measured $V_{c}$; Genzel et al. 2010). Even though these streaming motions are present, dynamical pressure is less important, according to the measured ratio $V_{c} / \sigma$.

\subsubsection{Comparison with Other Findings}

In our picture, shorter depletion times are associated with greater levels of turbulence and higher $\sigma$. This is also a feature of the model proposed by Renaud et al. (2012) based on feedbackregulated turbulence. The higher turbulence in $z=2$ mergers as compared with $z=2$ disks leads to a higher fraction of (locally) dense gas, higher overall SFR, and thus a vertical offset in $\Sigma_{\text {SFR }}$ versus $\Sigma_{\text {gas }}$ space. Despite consistency with this dependence of $\tau_{\text {dep }}$ on $\sigma$, though, we interpret the role of $\sigma$ differently. In the Renaud et al. (2012) model, lower levels of turbulence lead to less effective turbulent triggering of SF and longer depletion times. In our picture, a lower $\sigma$ leads to a more destabilized system and thus to the onset of streaming motions, which lengthen the depletion time. Note that both of these pictures appear to imply that SF consumes gas quicker when the gas is more stabilized (according to the increase in Toomre Q with the higher effective gas sound speed in the presence of greater turbulence), perhaps contrary to expectations.

Interestingly, at fixed $\sigma$, Equations (11) and (17) together predict that the gas depletion time increases nonlinearly with decreasing gas fraction, or that the SFE increases nonlinearly with gas fraction. In addition, more centrally concentrated gas, situated in the portion of the disk where the rotation curve is rising and $\beta \sim 1$, will have slightly longer $\tau_{\text {dep }}$ than more extended disks with $\beta=0$. In the former case, shear prevents gas from forming collapsing structures. Our picture is thus also able to describe the findings of Saintonge et al. (2012) and may prove descriptive, from an observational perspective, of "morphological quenching," as envisioned by Martig et al. (2009).

We can now put this all together to predict a dependence

$$
\log \Sigma_{\mathrm{SFR}}=\log \Sigma_{\mathrm{gas}}-\log t_{\mathrm{ff}} / \epsilon_{\mathrm{ff}}-\frac{(\beta+1)}{4 f_{g}}
$$

for a Toomre unstable disk $(Q \approx 1)$ and taking for simplicity $\Sigma / \Sigma_{0} \sim m=2$ and $\gamma=-1.5$ for the cloud mass-spectrum index (i.e., Rosolowsky 2005). Assuming a universal $t_{\mathrm{ff}} / \epsilon_{\mathrm{ff}}$ that sets the intercept of a "fiducial" linear relation between $\log \Sigma_{\text {SFR }}$ and $\Sigma_{\text {gas }}$, then systems will fall progressively below this line as their gas fractions decrease. This form of the SF relation can consistently describe the strong offsets between various populations in the standard KS plot, as assembled and presented by Krumholz et al. (2012). Systems with the highest gas fractions - high- $z$ starbursts, local starbursts, and even including star-forming clouds in the Milky Way—are offset to the shortest depletion times. Below these fall the high- $z$ star forming disks at intermediate $\tau_{\mathrm{dep}}$, followed by the local star forming galaxies at the longest $\tau_{\mathrm{dep}}$. 
Of course, streaming motions may not be responsible for the entirety of the offset in the observed gas depletion time both within and among galaxies. Some part of the offset can be attributed to, and removed by, variation in the CO-to$\mathrm{H}_{2}$ conversion factor $\left(X_{\mathrm{CO}}\right)$. The bimodality between local and high- $z$ systems is arguably enhanced due to the adoption of a bimodal $X_{\mathrm{CO}}$ (Narayanan et al. 2011). Allowing for a dependence of $X_{\mathrm{CO}}$ on metallicity, low-mass dwarfs (with high $\left.X_{\mathrm{CO}}\right)$ appear to have similar gas depletion times as more massive disks (Schruba et al. 2012; unlike in Figure 8 where a single $X_{\mathrm{CO}}$ is assumed).

On the other hand, it seems that not all depletion time variations between different populations can be minimized in this way. In M33, a spiral galaxy intermediate in mass and metallicity between the Milky Way and Local Group dwarfs, the depletion time is shorter than for spirals in the Kennicutt (1998) sample, even adopting a slightly higher-than-Galactic $X_{\mathrm{CO}}$ appropriate for its metallicity (Braine et al. 2011). In addition, although Leroy et al. (2013) find that most variation in $\tau_{\text {dep }}$ internal to galaxies can be accounted for by varying $X_{\mathrm{CO}}$, the differences in $X_{\mathrm{CO}}$ between the disks and central regions of galaxies measured by Sandstrom et al. (2013) in fact underscore the existence of two different "modes" of SF (Leroy et al. 2013).

\section{CONCLUDING REMARKS}

In this paper, we examined the influence of non-axisymmetric stellar structure (i.e., nuclear bar, spiral arms) on gas flows, molecular cloud properties, and SF in the inner disk of the iconic spiral galaxy M51. Leveraging our unique view of gas motions, which includes both measurements of present-day torques and non-circular streaming motions decomposed from the line-ofsight velocity field, we establish an anti-correlation between gas flows and strong SF in M51. To explain the observed gas flow and SF patterns, we developed a simple model in which dynamical pressure is a critical parameter for determining cloud stability against gravitational collapse. We report the following results and conclusions.

1. A radial profile of the stellar torques across the PAWS field reveals distinct dynamical zones within which gas is driven radially inward or outward in response to the non-axisymmetric structure present in M51's inner disk. In the nuclear bar region $\left(\lesssim 20^{\prime \prime}\right)$ and inside the corotation resonance of the first spiral arm $\left(35^{\prime \prime}<R<55^{\prime \prime}\right)$, gas is driven strongly radially inward. Elsewhere in the disk, gas is either stationary or experiences a radially outward torque.

2. We use a unique method to decompose the line-of-sight velocity field of the $\mathrm{CO}$ emission into its radial and azimuthal components. Across the PAWS field, the sign and magnitude of our derived radial gas streaming motions agree very well with our expectations for how the molecular ISM should respond to torquing by the non-axisymmetric stellar structure.

3. Comparison of the radial torque profile and gas streaming motions with the molecular gas surface density and SFR (as traced by the combination of $\mathrm{H} \alpha$ and $24 \mu \mathrm{m}$ emission) reveals a clear anti-correlation between strong gas flows and active high-mass SF. In M51, radially inflowing gas appears to be less efficiently forming stars. More generally, gas sitting near the corotation of the disk and the bar or spiral structure forms stars more efficiently than gas that is far from corotation and in motion relative to the background potential.
4. We propose that the complementary patterns of gas flow and SF in the PAWS field are evidence for the importance of external pressure on the properties and stability of starforming clouds in M51. More precisely, we develop a simple model based on Bernoulli's principle that describes changes in cloud surface pressure in the presence of strong streaming motions (dynamical pressure). Such changes in surface pressure can make the difference between stability and collapse when, as in M51's spiral arms, clouds are dynamically coupled to their environment (i.e., when the cloud internal and external pressures are similar). In M51, cloud stabilization occurs preferentially as the result of strong radial inflow motions in regions of negative torque, but we expect that outflow motions may serve the same role in other systems.

5. Our model successfully reproduces several key observations. We can retrieve the environment-dependent variation of the slope of the cloud mass spectrum measured directly from the cloud distribution. We can also explain the overall difference in the masses of clouds between the arm and interarm. Sensitivity to changes in dynamical pressure are also consistent with the observation that not all of M51's clouds are virialized, as evidenced by the lack of a clear size-linewidth relation for clouds in M51.

6. We investigate other potential sources of cloud stability (shear, galactic shocks, stellar feedback-driven turbulence) and find that they cannot uniformly explain the observed non-monotonic radial dependence of the gas depletion time. The cloud formation mechanism also has little influence on the ability of clouds in M51 to collapse and form stars: while cloud collision and agglomeration in the spiral shock are likely present, we find no obvious relation to SF in M51's current gas reservoir.

7. Although non-circular motions in M51 are particularly high, the influence of dynamical pressure should be commonplace in spiral galaxies. We suggest that gas flows within galaxies are a source of scatter in the KS SF relation both within and among galaxies. Late-type spiral disks with tightly wound spirals that drive weak streaming motions will form their stars at a faster rate per unit gas mass than early-type disks where streaming motions are higher.

8. We propose that our model for the dependence of gas depletion time on dynamical pressure links low- and high- $z$ SF from dense, molecular material. In particular, we suggest that systems form stars with progressively shorter depletion times as their gas fractions increase.

We thank the IRAM staff for their support during the observations with the Plateau de Bure interferometer and the $30 \mathrm{~m}$ telescope. D.C. and A.H. acknowledge funding from the Deutsche Forschungsgemeinschaft (DFG) via grant SCHI 536/5-1 and SCHI 536/7-1 as part of the priority program SPP 1573 "ISMSPP: Physics of the Interstellar Medium.” C.L.D. acknowledges funding from the European Research Council for the FP7 ERC starting grant project LOCALSTAR. T.A.T. acknowledges support from NASA grant No. NNX10AD01G. During this work, J. Pety was partially funded by the grant ANR-09-BLAN-023101 from the French Agence Nationale de la Recherche as part of the SCHISM project (http://schism.ens.fr/). E.S., A.H., and D.C. thank the NRAO for their support and hospitality during their visits in Charlottesville. E.S. thanks the Aspen Center for Physics and NSF grant No. 1066293 for hospitality 


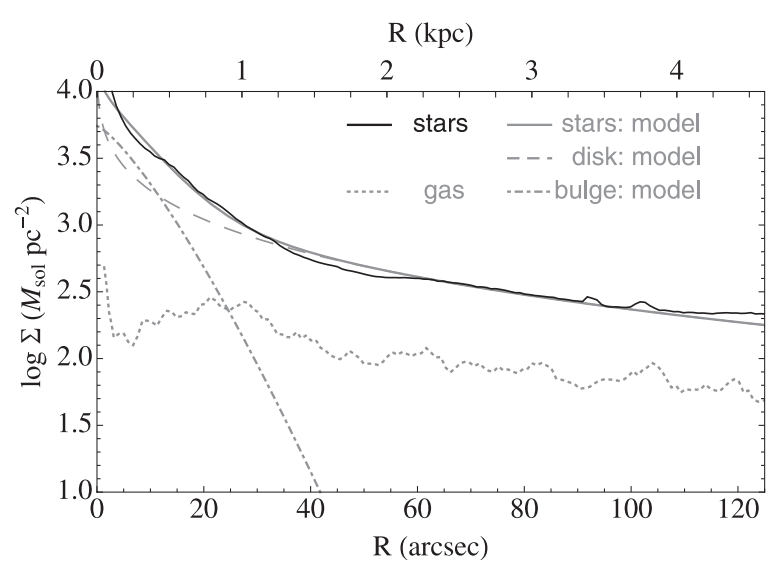

Figure 9. Surface density profiles in the central $9 \mathrm{kpc}$ of M51. The thin gray dotted line shows the total gas surface density $\Sigma_{\text {gas }}=\Sigma_{\mathrm{H}_{2}}+\Sigma_{\mathrm{H}_{\mathrm{I}}}$. The solid gray and black lines show the modeled and observed stellar surface density $\Sigma_{\star}$ assuming the best-fit stellar $M_{\star} / L_{3.6}$ (determined below). The model components are shown in thin gray lines: exponential stellar disk (dashed) and Gaussian bulge (dot-dashed).

during the development and writing of this paper. S.G.B. acknowledges economic support from Junta de Andalucia grant P08 TIC 03531. The National Radio Astronomy Observatory is a facility of the National Science Foundation operated under cooperative agreement by Associated Universities, Inc.

\section{APPENDIX A}

\section{MASS-BASED ROTATION CURVE FOR M51}

We use the standard approach to assemble an estimate of the rotation curve in M51 by summing the individual contributions of the stellar and gaseous components to the circular velocity, adopting a simple mass-follows-light model appropriate for spiral galaxies (i.e., the rotation curve can be accounted for with baryons alone). ${ }^{20}$

This requires first constructing radial profiles of the stellar and gas surface densities. The total gas surface density $\Sigma_{\text {gas }}$ is assembled from measurements of $\Sigma_{\mathrm{H}_{1}}$ and $\Sigma_{\mathrm{H}_{2}}$ traced by the THINGS H i zeroth-moment map (Walter et al. 2008) and PAWS CO(1-0) observations (Pety et al. 2013), respectively, and includes a factor of 1.36 to account for the presence of helium. As in Section 2, we assume a constant CO-to- $\mathrm{H}_{2}$ conversion factor of $X_{\mathrm{CO}}=2 \times 10^{20} \mathrm{~cm}^{-2}\left(\mathrm{~K} \mathrm{~km} \mathrm{~s}^{-1}\right)^{-1}$ and also include a sky-to-disk plane correction of $\cos 21^{\circ}$.

The stellar surface density $\Sigma_{\star}$ is traced by the $\mathrm{S}^{4} \mathrm{G} 3.6 \mu \mathrm{m}$ map of the old stellar light (corrected for non-stellar emission; Meidt et al. 2012). The (assumed) global $3.6 \mu \mathrm{m}$ mass-to-light ratio $\Upsilon_{3.6}$ (with which $\Sigma_{\star}=\Upsilon_{3.6} L_{3.6} \cos 21^{\circ}$ ) is the single free parameter to be fit in comparing the modeled and observed circular velocities. The final surface density profiles are shown in Figure 9. Below, we adopt models of the individual exponential disk and Gaussian bulge components of the stellar surface brightness.

To estimate the circular velocities associated with the gaseous and stellar disks, we adopt the thin disk approximation and model the bulge separately. The circular velocity at radius $R$ (in the plane of the disk) is given by

\footnotetext{
20 The maximum disk hypothesis has been found to yield good fits inside the optical radius of $3 / 4$ of the galaxies in a spiral sample studied by Palunas \& Williams (2000).
}

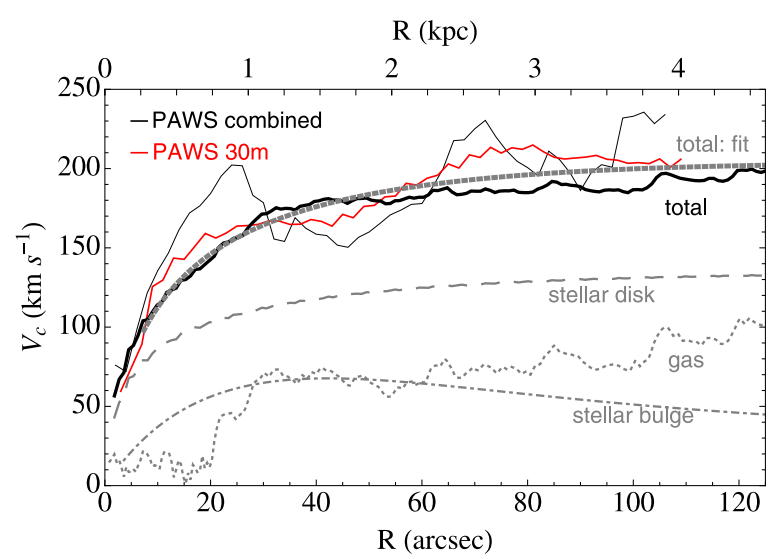

Figure 10. Rotation curves in the center $(R<4.5 \mathrm{kpc})$ of M51. Circular velocities derived from the PAWS $\mathrm{CO}(1-0)$ data are shown in thin lines (combined data, black; single-dish, red; errors are omitted for clarity). The thick solid black line shows the total $V_{c}$, calculated as the sum of the individual modeled circular velocities shown in thin gray lines: stellar bulge component (dot-dashed), stellar disk component (dashed), gas (dotted). Comparing the red curve with our smooth fit with the total circular velocity (thick gray dotted line) gives a best-fit $M / L=0.45 \pm 0.15$.

$$
V_{c, D}^{2}(R)=2 \pi G R \int_{0}^{\infty} \int_{0}^{\infty} \Sigma\left(R^{\prime}\right) J_{0}\left(k R^{\prime}\right) J_{1}(k R) R^{\prime} k d k d R^{\prime},
$$

where $J_{0}$ and $J_{1}$ are cylindrical Bessel functions (e.g., Barnes \& Sellwood 2003; Barnes et al. 2004). Without accounting for disk thickness, which would reduce the circular velocity, the derived $\Upsilon$ is slightly underestimated.

Following Palunas \& Williams (2000), we estimate the contribution from the stellar bulge to $V_{c}$ by modeling the bulge component of the stellar surface brightness profile with a multiGaussian expansion

$$
\mu_{B}\left(a_{B}\right)=\sqrt{\frac{f}{\pi}} \sum_{k=1}^{n} \frac{c_{k}}{R_{B_{k}}} e^{-a_{B}^{2} / R_{B_{k}}^{2}}
$$

where $a_{B}=R^{2}+\left(z /\left(1-\epsilon_{B}\right)\right)^{2}$ is the bulge semi-major axis length, $\epsilon_{B}=1-q_{p, B}$ is the bulge ellipticity, $q_{p, B}$ is the apparent bulge axis ratio, $f=\cos i^{2}+\sin i^{2} / q_{B}^{2}$, and, for each of $k$ components, $c_{k}$ is the total light and $R_{B_{k}}$ is the scale length.

This 2D elliptical Gaussian distribution deprojects to a $3 \mathrm{D}$ spheroidal distribution with intrinsic axis ratio $q_{B}=\left(q_{p, B}^{2}-\right.$ $\left.\cos i^{2}\right) / \sin i^{2}$, whereby (in the plane of the disk)

$$
V_{c, B}^{2}(R)=4 \pi G \Upsilon_{3.6} \sqrt{1-e_{B}^{2}} \int_{0}^{R} \frac{\mu\left(R^{\prime 2}\right) R^{\prime 2} d R^{\prime}}{\sqrt{R^{2}-e_{B}^{2} R^{\prime 2}}},
$$

where $e_{B}$ is the bulge eccentricity, which we take to be zero for simplicity (given the small degree of asymmetry in the central projected light distribution). We expect this assumption to have less consequence than our requirement for a global $\Upsilon_{3.6}$, uniform from bulge to disk.

Modeled circular velocities are plotted in Figure 10 together with two independent estimates of the rotational velocity measured from the PAWS $\mathrm{CO}(1-0)$ observations (Colombo et al. 2013a). The thick black curve shows the rotational velocity measured by fitting tilted rings to the line-of-sight velocities in the combined interferometric and single-dish data (with native $1^{\prime \prime}$ resolution; Pety et al. 2013) using the GIPSY task ROTCUR with fixed center, P.A. $=173^{\circ}$, and inclination angle $i=21^{\circ}$. 
The thick red curve shows the rotational velocity measured from the $30 \mathrm{~m}$ single-dish data (12" resolution) assuming the same kinematic parameters. We expect this curve to be a fairer representation of the true circular velocity, as beam smearing at lower resolution minimizes the contribution from non-circular streaming motions to the measured rotational velocity (evident as wiggles in the thin curve from the higher-resolution data). Our optimal $\Upsilon_{3.6}$ is chosen based on agreement with this estimate of the circular velocity, as described below. We adopt a smooth three-parameter fit to the total velocity (i.e., Faber \& Gallagher 1979) as our final model for $V_{c}$. With our mass-follows-light model, we find a best-fit stellar $\Upsilon_{3.6}=0.45 \pm 0.15$. This value agrees well with the value $\Upsilon_{3.6}=0.5$ measured by Eskew et al. (2012), which is itself very near the value that has been previously assumed to convert between $3.6 \mu \mathrm{m}$ luminosity and mass (Leroy et al. 2008). The maximum disk hypothesis is evidently reliable in this case, at least throughout the PAWS field of view.

\section{APPENDIX B}

\section{DEFINING THE DYNAMICAL ENVIRONMENT: PRESENT-DAY TORQUES}

The impact of environment is underlined by present-day torques exerted by non-axisymmetric structure in the disk. We use information supplied by these gravitational torques to define the dynamical environment.

\section{B.1. Gravitational Torque Estimation}

The $S^{4} \mathrm{G} 3.6 \mu \mathrm{m}$ map of the old stellar light (Meidt et al. 2012; from which a contribution from non-stellar emission has been removed) presents us with an optimal view of the backbone of present-day gravitational torques. ${ }^{21}$ To generate a map of the stellar potential, we first deproject the image according to the inclination $i=21^{\circ}$ and major axis P.A. $=173^{\circ}$ determined by Colombo et al. (2013b) from gas kinematics. We then apply a constant stellar mass-to-light ratio $(M / L)$ to the $3.6 \mu \mathrm{m}$ image and map this into density by assuming a simple tanh vertical distribution with uniform scale height. Like the stellar $M / L$, the adopted vertical distribution will affect the magnitude of the measured torques but not the radial dependence highlighted below, which is our primary concern. Triaxiality in the dark matter halo could potentially introduce a change in the radial behavior, but we expect this to be minimal over our relatively small and centralized field of view covering the inner $9 \mathrm{kpc}$. The dominance of the stellar mass over this area is confirmed by our study of gas kinematics in Section 3.2, which presents supporting evidence for the conclusions drawn from the stellar potential alone.

From the potential, we calculate a map of the quantity $-R \times$ $\nabla \Phi$ (Figure 11) representing the inertial torque per unit gas mass, following Garcia-Burillo et al. (2005, 2009) and Haan et al. (2009). In the rotating (non-inertial) frame, forcing includes a contribution from the "fictitious" centrifugal and Coriolis terms. Since the cross product of the radius $R$ with these terms goes identically to zero in the former case and to zero with averaging over $0-2 \pi$ in azimuth in the latter, then a measure of the time average of the rate of change of the angular momentum, the torque, can be obtained by taking azimuthal averages of $R \times \nabla \Phi$, with weighting by the molecular gas surface density.

\footnotetext{
21 Note that the influence of the companion is included to the extent that it has contributed to the reorganization of the stellar mass and structure in the disk mapped in the stellar potential.
}

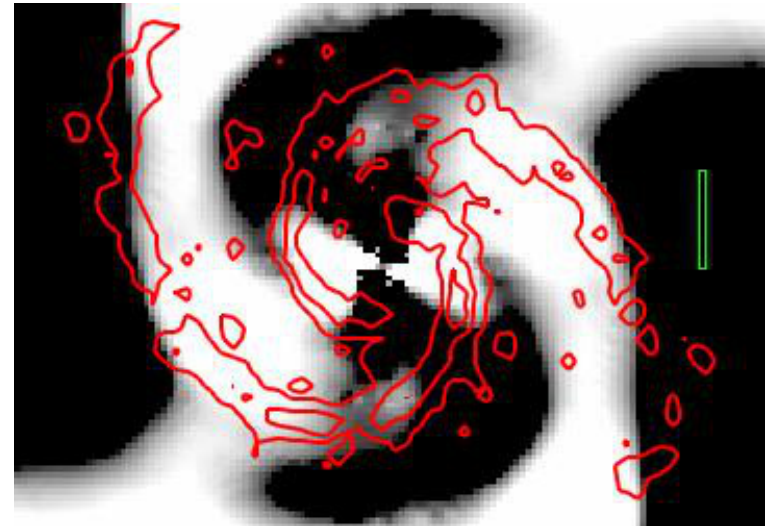

Figure 11. Map of torques in the inertial frame $R \times \nabla \Phi$, generated from the $3.6 \mu \mathrm{m}$ map of the old stellar light tracing the stellar potential $\Phi$ (see the text). White (black) corresponds to positive (negative) torques that drive motions radially outward (inward). Contours of the CO intensity are overlaid in red. The green bar at right indicates $40^{\prime \prime}$. Image dimensions: $\sim 300^{\prime \prime} \times 200^{\prime \prime}$.

This is equivalent to the approach of Garcia-Burillo et al. (2005) and Haan et al. (2009), where the molecular gas surface density represents the probability of finding the gas at its current position at present. Here, we use the PAWS observations of $\mathrm{CO}(1-0)$ emission to trace the molecular gas surface density assuming a CO-to- $\mathrm{H}_{2}$ conversion factor $X_{\mathrm{CO}}=2 \times 10^{20} \mathrm{~cm}^{-2}\left(\mathrm{~K} \mathrm{~km} \mathrm{~s}^{-1}\right)^{-1}$ (Colombo et al. 2013a). These observations include single-dish data, which have been found to be a critical addition to the gas tracers used for this purpose (Garcia-Burillo et al. 2005; van der Laan et al. 2011); extended emission on the largest scales must be included for accurate determination of the net torque. Note that the assumed conversion factor is another potential influence on the magnitude of the derived torques. For this reason, in the upcoming section, we present the torque profile in units of the net torque over the PAWS field of view.

\section{B.2. Inventory of Distinct Dynamical Environments}

Figure 1 in the main text shows the radial profile of azimuthally averaged torques across the PAWS field of view. Each crossing from negative to positive marks a $\mathrm{CR}$, while crossings from positive to negative torque coincide with a switch in predominance to a new pattern. Three dynamically distinct zones are identified, as described below.

The first CR in Figure 1 occurs just outside the "butterfly" pattern in the central $20^{\prime \prime}$ of the torque map, which is characteristic of the bar influence (i.e., Garcia-Burillo et al. 1993). Comparison with the velocity field confirms that the gas is responding to torquing by the stellar bar (first identified in the near-infrared by Zaritsky et al. 1993), even though the stellar bar lacks a molecular counterpart. The bar is responsible for the $+10^{\circ}-15^{\circ}$ twist in the orientation of the line of nodes, previously interpreted as evidence for variation in the galaxy major axis tracing a warp or twist of the disk (Shetty et al. 2007). This marks the first evidence that the bar, rather than the spiral, dominates the kinematics in the central $30^{\prime \prime}$.

The influence of the bar also extends to gas morphology, as evidenced by the build-up of a molecular ring at the overlap with the molecular spiral arms just exterior, near $R=35^{\prime \prime}$. Opposing torques from inside the ring, where the bar drives gas outward, and outside the ring, where torques drive gas inward (see below), result in the pile-up of gas at the location of the ring.

Beyond the second $\mathrm{CR}$ at $R_{\mathrm{CR}}=55^{\prime \prime}$, gas is again driven outward, across the outer zone of the CO-bright spiral arms. 


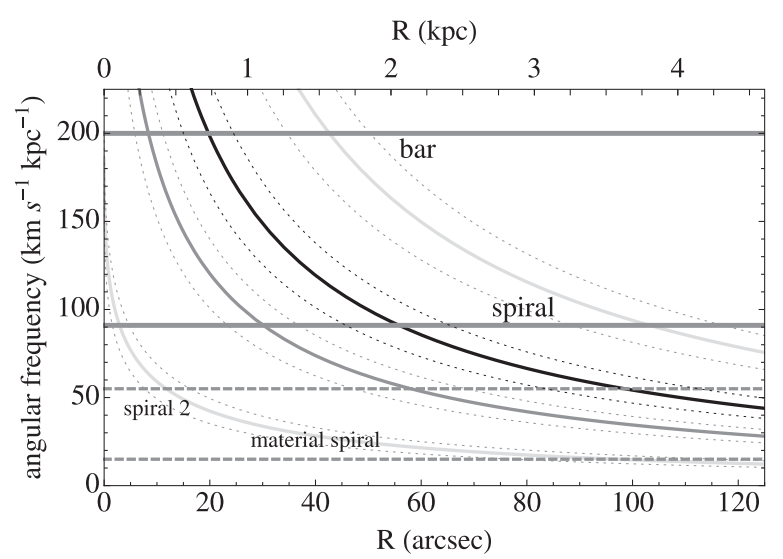

Figure 12. Pattern speed estimates for structure in the disk of M51. Angular frequency curves are shown: $\Omega$ (black), $\Omega-\kappa / 4$ (gray), and $\Omega \pm \kappa / 2$ (light gray). Estimates for the bar and spiral pattern speeds implied by the CRs revealed by the torque profile in Figure 1 are shown as gray horizontal lines. A possible second spiral speed (see the text for a discussion) and the rotation of the material arms are also indicated by gray horizontal dashed lines.

Radial outflow continues until the start of the third distinct pattern, near $R=85^{\prime \prime}$, where the direction of flow is again reversed. This location is consistent with previous estimates for the start of the distinct outer, material spiral pattern (e.g., Tully 1974; Vogel et al. 1993; Meidt et al. 2008b), which continues beyond the edge of our field of view.

In total, the gravitational torques expose three dynamically distinct zones dominated by at least three unique nonaxisymmetric structures. The number, multiplicity, and radial domains of patterns present in the disk will be the subject of a more detailed study in the future (Colombo et al. 2013b). Below, we briefly review supporting evidence for our identification of these three main environments.

\section{B.2.1. Complementary Evidence for Multiple Patterns}

We expect at least three distinct pattern speeds in the central $9 \mathrm{kpc}$ of M51, coinciding with three distinct structures: the bar, the main spiral, and an outer spiral. For the main spiral pattern with corotation at $R_{\mathrm{CR}}=55^{\prime \prime}$, we estimate a pattern speed of $\Omega_{p} \sim 90 \mathrm{~km} \mathrm{~s}^{-1} \mathrm{kpc}^{-1}$ based on intersection with our adopted angular frequency curve (see Figure 12). This is very close to the value measured by Meidt et al. (2008b) with the radial Tremaine-Weinberg (TWR; Merrifield et al. 2006, Meidt et al. 2008a) method using lower-resolution molecular gas observations as a kinematic tracer and assuming a similar major axis P.A. $=175^{\circ} \cdot 22,23$

At smaller radii, the gravitational torques suggest a transition to a distinct bar pattern, with $R_{\mathrm{CR}}=20^{\prime \prime}$, which is comparable to

\footnotetext{
22 Accounting for differences in adopted distance and inclination angle.

23 Two speeds from an alternatively plausible TWR solution, which assumes a lower P.A. $=170^{\circ}$, straddle our adopted value $\Omega_{p} \sim 90 \mathrm{~km} \mathrm{~s}^{-1} \mathrm{kpc}^{-1}$. Based on evidence here for the bar influence on morphology and molecular gas kinematics (see Section 3.2), though, it seems likely that the higher inner pattern speed measured by Meidt et al. (2008b) reflects a combination of the speed at $\Omega_{p}=90 \mathrm{~km} \mathrm{~s}^{-1} \mathrm{kpc}^{-1}$ and a yet higher bar pattern speed. This circumstance has been encountered in other galaxies (Meidt et al. 2009), where the resolution in the TWR solution was limited by that of the kinematic tracer. According to our estimate of the bar CR, the bar zone would have been probed by only the inner three to four $\sim 6^{\prime \prime}$ radial bins used by Meidt et al. (2008b). In addition, given the orientation of twisted line-of-nodes manifest by the bar, the higher P.A. $=175^{\circ}$ major axis orientation for the TWR solution presumably disfavors the detection of the bar influence, more so than for a lower assumed P.A.; as for the TWR solution at P.A. $=170^{\circ}$, a third solution with P.A. $=165^{\circ}$ measured by Meidt et al. (2008b) suggests an inner pattern speed $>90 \mathrm{~km} \mathrm{~s}^{-1}$ $\mathrm{kpc}^{-1}$.
}

the location recently suggested by Zhang \& Buta (2012). From this, we estimate a bar pattern speed of $\Omega_{b} \sim 200 \mathrm{~km} \mathrm{~s}^{-1} \mathrm{kpc}^{-1}$. In this case, the bar and spiral pattern speeds offer a physically plausible scenario, as the corotation of the bar coincides with the inner 4:1 resonance of the spiral. Resonance overlaps of this kind have been identified in barred spiral simulations (Rautiainen \& Salo 1999; Debattista et al. 2006; Minchev et al. 2011) and may be characteristic of nonlinear mode coupling (e.g., Tagger et al. 1987; Sygnet et al. 1988), whereby energy and angular momentum are transferred between the modes. Note that with this set of pattern speeds, the molecular ring sits near the spiral's inner 4:1 resonance (and near the bar's outer 4:1 resonance) and forms at the intersection of positive torquing by the bar outside its $\mathrm{CR}$ with the negative torquing by the spiral inside its CR. The inner 4:1 resonance has previously been suggested to favor the population of gas in the form of such a ring (Garcia-Burillo et al. 2009). (We also note that the coincidence of the end of the bar with its CR makes this bar a so-called "fast" bar.)

This same process could be at work further out in the disk, in the zone between $35^{\prime \prime}$ and $70^{\prime \prime}$. At its $R_{\mathrm{CR}}=55^{\prime \prime}$, the speed $\Omega_{p}=$ $90 \mathrm{~km} \mathrm{~s}^{-1} \mathrm{kpc}^{-1}$ could overlap with the inner $4: 1$ resonance of a lower speed of $\Omega_{p, 2} \sim 55 \mathrm{~km} \mathrm{~s}^{-1} \mathrm{kpc}^{-1}$ (see Figure 12), just before the start of the material arms. This second spiral speed falls just within the range of speeds suggested for a similar second speed in the TWR solution at $170^{\circ}$ measured by Meidt et al. (2008b).

The existence of this second more slowly rotating pattern may be plausible for several reasons. (1) The gas kinematics less clearly show the pattern of outflow in the post-corotation zone $55^{\prime \prime}<R<70^{\prime \prime}$, as would be expected for a pattern outside its CR. ${ }^{24}$ Two patterns, each propagating inside their CR, would maintain radial inflow through this zone. (2) The spatial offset between young star and gas tracers does not clearly switch sign across the corotation at $R_{\mathrm{CR}}=55^{\prime \prime}$, as predicted in response to a spiral density wave around CR. Again, two patterns, both propagating inside the $\mathrm{CR}$ and both driving gas radially inward, remove the possibility that young stars will be found upstream of the gaseous spiral, as otherwise expected for a single pattern outside its CR. (3) While the $3.6 \mu \mathrm{m}$ map of old stellar light shows a pronounced $m=2$ perturbation, this is identifiable as a two-armed spiral only outside $R \sim 55^{\prime \prime}$. Inside this radius, in the zone $35^{\prime \prime}<R<55^{\prime \prime}$, ellipse fits to $3.6 \mu \mathrm{m}$ isophotes reveal a more oval, bar-like structure (see also Schinnerer et al. 2013). Other stellar traces (from FUV to $\mathrm{H} \alpha$ ) reveal a similarly marked absence of a clear spiral pattern in this zone. In this case, the higher pattern speed of $\Omega_{p} \sim 90 \mathrm{~km} \mathrm{~s}^{-1} \mathrm{kpc}^{-1}$ might apply to a bar terminating at its $\mathrm{CR}$, while the lower pattern speed $\Omega_{p, 2} \sim 55 \mathrm{~km} \mathrm{~s}^{-1} \mathrm{kpc}^{-1}$ could describe the strong spiral pattern. We might then interpret the gaseous response to the oval perturbation, which is very definitely in the form of a two-armed spiral, as equivalent to dust lanes running along the length of a stellar bar. Alternatively, the main spiral pattern could overlap with a lower, outer pattern with speed $\Omega_{p}=20 \mathrm{~km} \mathrm{~s}^{-1} \mathrm{kpc}^{-1}$ near where its outer Lindblad resonance and the outer pattern's inner Lindblad resonance coincide (see Figure 12).

For simplicity in what follows, we describe the dynamical environment by three distinct zones dominated by an inner, or

\footnotetext{
24 The radial torque profile $\langle\Gamma\rangle(R)$ (Figure 1) suggests that radial gas inflow stops at $R_{\mathrm{CR}}=55^{\prime \prime}$, rather than continuing until $R=70^{\prime \prime}$, as suggested by the radial streaming motions $v_{r}$, measured in Appendix $\mathrm{C}$. This difference could be alleviated by a constant offset to either $v_{r}$ or $\langle\Gamma\rangle$ (with an impact on the locations of the other CRs, as well), or it could indicate a more complicated gaseous response to the torquing.
} 

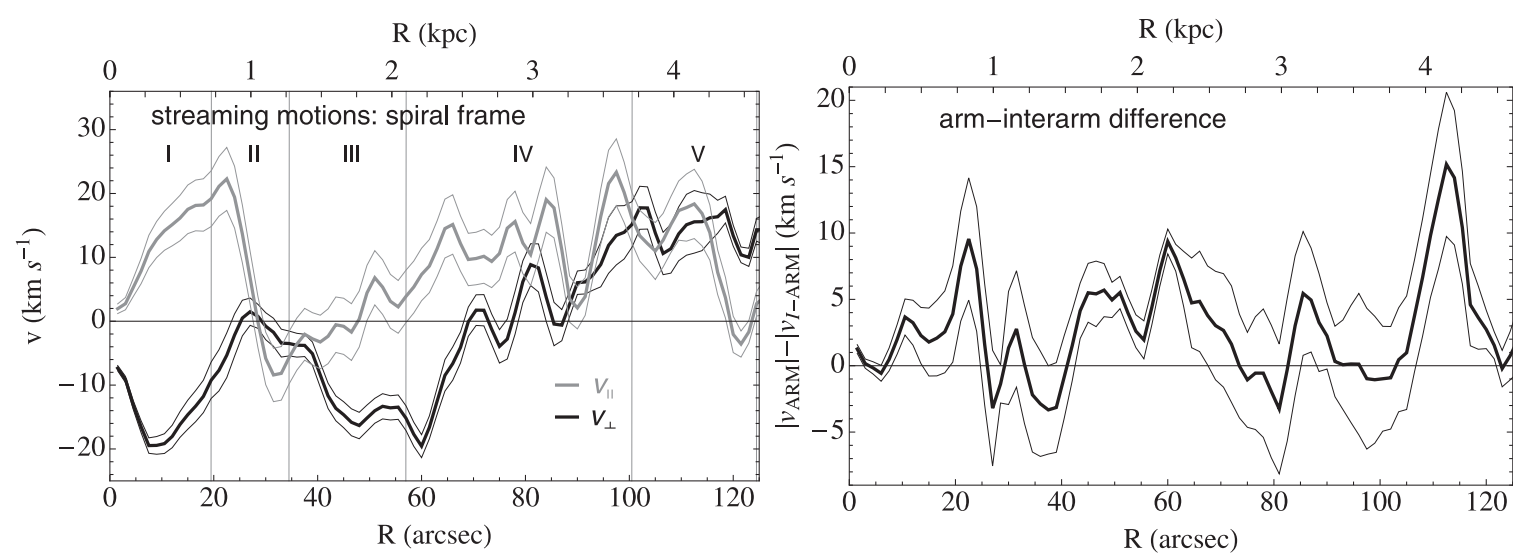

Figure 13. (Left) CO intensity-weighted velocities parallel ( $v_{\|}$, blue) and perpendicular $\left(v_{\perp}\right.$, red) to the spiral arm, calculated in radial bins as described in the text from the reconstructed radial and azimuthal velocities (see Figure 2). Dashed lines represent the uncertainty associated with each velocity component, calculated from the errors shown in Figure 2 (i.e., the rms dispersion in solutions with $\pm 5^{\circ}$ variation in the assumed spiral arm pitch angle $i_{p}$ and the uncertainty introduced by our adopted rotation curve). The intensity-weighting focuses our measurements on the CO-bright spiral arms. (Right) The difference in the magnitude of non-circular streaming motions in the arm and interarm.

nuclear, bar and two (inner and outer) molecular spirals. As explored in Section 3.2, the observed gas motions reflect the predicted combination of radial inflow and outflow that, in the spiral arm region, correspond to flow along and through the arm.

\section{APPENDIX C \\ DECOMPOSING THE PAWS LINE-OF-SIGHT VELOCITY FIELD}

The nearly logarithmic spiral in M51, spanning over $5 \mathrm{kpc}$ in radius, presents a unique opportunity to decompose the line-ofsight velocity

$$
\begin{aligned}
V_{\mathrm{los}}(R, \theta)= & V_{\mathrm{sys}}+\left[\left(V_{c}(R)+v_{\phi}(R, \theta)\right) \sin (\theta)\right. \\
& \left.+v_{r}(R, \theta) \cos (\theta)\right] \sin i
\end{aligned}
$$

into its radial and azimuthal components $v_{r}$ and $v_{\phi}$. Here, $V_{\text {sys }}$ is galaxy systemic velocity, $V_{c}$ is the disk circular velocity, and $\theta$ is measured with respect to the line of nodes. Following Colombo et al. (2013b), we adopt an inclination of $i=21^{\circ}$ and a major axis $\theta_{\text {P.A. }}=173^{\circ}$.

The key to decomposing $V_{\text {los }}$ is the idealized arm frame in which streaming motions $v_{r}$ and $v_{\phi}$ will be nearly constant in small segments at constant arm phase $\psi$ parallel to each arm. Adopting a spiral arm pitch angle of $i_{p}=21^{\circ}$, we transform from Cartesian to log-polar coordinates and define a grid of rectangular segments or bins aligned with the spiral arm. In each bin, we solve for $v_{r}$ and $v_{\phi}$ via simple matrix inversion, according to

$$
\left(V_{\text {los }}-V_{\text {sys }}\right) / \sin i-V_{c} \sin (\theta)=v_{r}(\psi) \cos (\theta)+v_{\phi}(\psi) \sin (\theta),
$$

where the coefficients are specified by the geometric factors.

We calculate maps assuming three different pitch angles: a nominal $i_{p}=21^{\circ}$ (i.e., Shetty et al. 2007) and a second set offset by $\pm 5^{\circ}$. The average of the solutions from these three cases define our estimate for each component, while the dispersion in these values supplies a measure of our uncertainty, together with the uncertainty introduced by our adopted rotation curve (see Appendix A). To accommodate for real variation in the molecular spiral arm pitch angle (determined to be $5^{\circ}$ between the arms and as much as $20^{\circ}$ along the arms by Patrikeev et al.
2006), we size the spiral segments over which the solution is calculated to be 5.6 wide and $\sim \log r\left({ }^{\prime \prime}\right)=0.011$. These segments cover a smaller area toward the center, so the solution in the bar zone, where a logarithmic-polar deprojection may be less fitting, should nevertheless be accurate.

Prior to obtaining the solution, the contribution of the circular velocity to the observed line-of-sight velocity field is removed (see Equation (C2)). We adopt the circular velocity estimated from our 1D mass model of M51, constructed as described in Appendix A. By using a mass-based reconstruction of the circular velocity, we avoid the uncertainties typically inherited by circular velocity estimates made directly from the lineof-sight velocity field (e.g., with tilted-ring fits), namely the contribution of azimuthal streaming motions. The result is a much more smooth, physically motivated model of the circular velocity curve that should insure accurate streaming motion solutions. We use the uncertainty introduced in the rotation curve by our choice of stellar $M_{\star} / L_{3.6}=0.45 \pm 0.15$ (Appendix A) to define the error in our measured streaming velocities, together with the measured pitch angle uncertainty. Both are typically $<25 \%$.

The tangential and radial streaming motions (shown in Figure 2 in the main text) translate into motion directed along and through the arm, channeled by the stellar spiral. This is depicted more clearly in the top left of Figure 13, showing intensity-weighted averages of the velocities in the spiral arm frame, assuming $i_{p}=21^{\circ}$. Here

$$
\begin{aligned}
v_{\perp} & =v_{r} \cos \left(i_{p}\right)+v_{\phi} \sin \left(i_{p}\right), \\
v_{\|} & =-v_{r} \sin \left(i_{p}\right)+v_{\phi} \cos \left(i_{p}\right),
\end{aligned}
$$

where $v_{\perp}$ runs perpendicular to the arm and $v_{\|}$runs parallel to the arm. Note that in the zone $40^{\prime \prime}<r<60^{\prime \prime}$, the velocities $v_{\perp}$ perpendicular to the spiral arm arise almost completely with the radial motions since the azimuthal streaming in this zone is at a minimum. The spiral arm pitch angle is meanwhile increasing here, by up to $30^{\circ}$, so that the radial streaming motions could be distributed slightly more in the direction parallel to the spiral. This would lead to larger $v_{\|}$than measured here (assuming $i_{p}=21^{\circ}$ ), although the perpendicular motions still dominate in this case.

As described in Section 3.2, we use CO intensity as a weighting factor in binned azimuthal averages to reveal the 

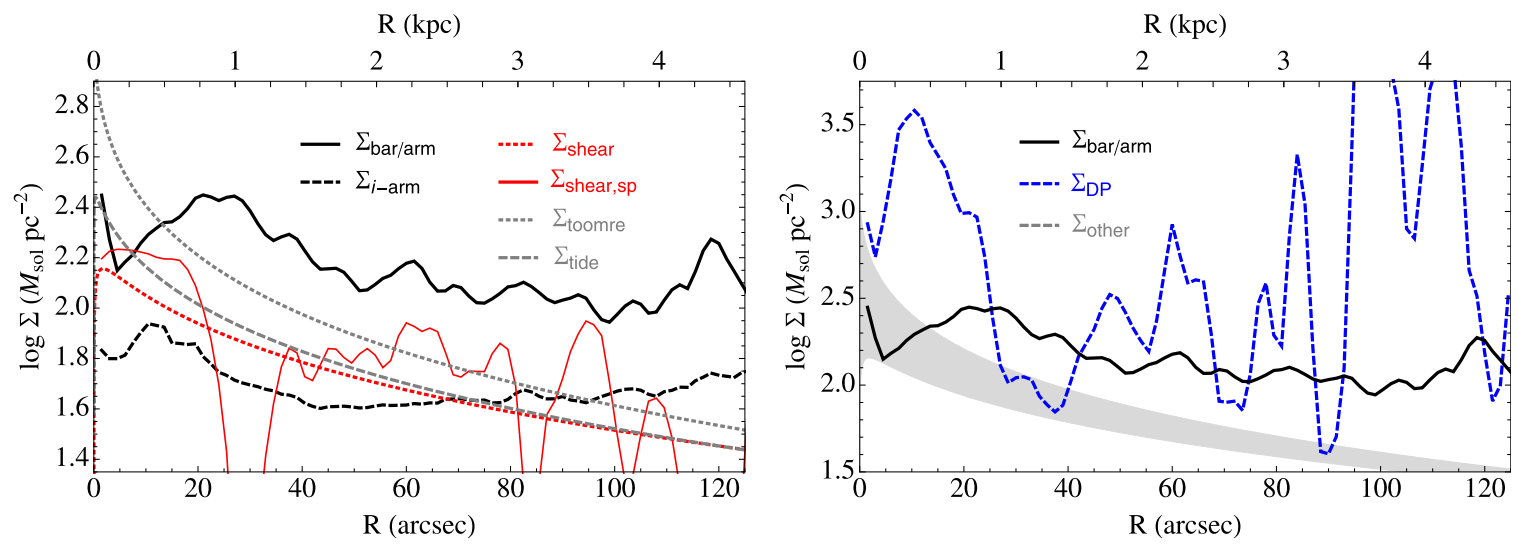

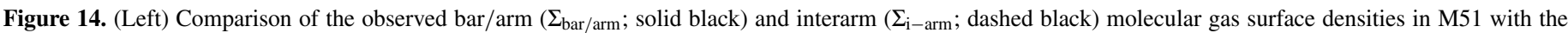

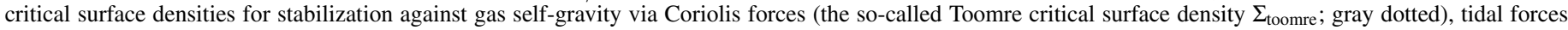

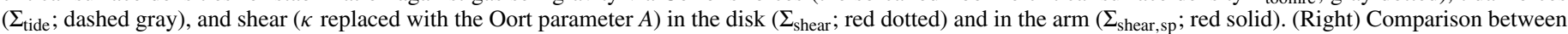

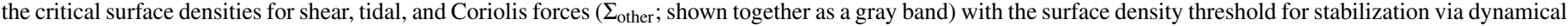
pressure $\left(\Sigma_{\mathrm{DP}}\right.$; dashed blue). The observed bar/arm surface density ( $\Sigma_{\text {bar/arm }}$; solid black) is repeated from the left panel.

velocities characteristic of the (CO-bright) spiral arms. Profiles with inverse-intensity weighting, which highlight the interarm streaming velocities, are qualitatively similar but show velocities $\approx 5-10 \mathrm{~km} \mathrm{~s}^{-1}$ lower than the profiles in Figure 2 . The difference in the magnitudes of streaming motions in the arm and interarm is shown in the right panel of Figure 13.

\section{APPENDIX D}

\section{GRAVITATIONAL DISK STABILITY}

In this section, we examine in greater detail the stability of the molecule-dominated gas disk in M51. We quantify and compare the critical surface density for dynamical pressure to that in the presence of shear, Coriolis forces, and tidal forces, three sources of cloud stability of potential consequence for the global pattern of SF.

Following Toomre (1964), we take

$$
\Sigma_{\text {toomre }}=\frac{\alpha \sigma \kappa}{\pi G}
$$

as defining the threshold for stability in a thin rotating gas disk, whereby the Toomre $Q=1$ when $\Sigma_{\text {toomre }}=\Sigma_{\text {gas }}$. Here, $\sigma$ is the gas velocity dispersion, $\kappa$ is the epicyclic frequency measured from our rotation curve model (see Appendix A), and $\alpha$ is a dimensionless factor calibrated empirically. ${ }^{25}$ (In the following, we let $\alpha=1$ but also discuss the impact of variation in this value.) Below this threshold, Coriolis forces spin up the gas so that centrifugal forces counter the self-gravity of the gas, resulting in a suppression of cloud (and star) formation. Above the threshold, gas is unstable to large-scale collapse, leading to SF.

The threshold for cloud stability in the presence of shear, as derived by Elmegreen (1993; see Luna et al. 2006), can be written as

$$
\Sigma_{\text {shear }}=\frac{2.5 \alpha_{A} \sigma A}{\pi G}
$$

\footnotetext{
25 Values $\alpha \neq 1$ are chosen in order that $Q=1$ at the location of the observed SF threshold. As suggested by Schaye (2004) and de Blok \& Walter (2006), deviations in $\alpha$ from unity may be commonly required only because measurements of the gas velocity dispersion from observations of the atomic phase systematically overestimate the true $\sigma$ of gas in a cold phase, which we can better approximate with the PAWS observations (as confirmed in M51 by Colombo et al. 2013b).
}

where $A$ is the Oort parameter and again we set the dimensionless factor $\alpha_{A}$ to unity. We consider separately the background shear due to differential rotation and the spiral arm shear, accounting for gradients in the azimuthal spiral arm streaming in the definition of $A$ (in addition to background shear), as in Section 5.1.1 in the main text.

Alternatively, cloud shearing via tidal forces will become ineffective above the threshold

$$
\Sigma_{\text {tide }}=\frac{\sigma[3 A(A-B)]^{1 / 2}}{\pi G},
$$

following Kenney et al. (1993), with Oort $B=\Omega-A$. Note that these formulations of cloud stability ignore any modulation/ dissipation of shear, tidal, or Coriolis forces due to additional effects, such as magnetic fields or viscosity.

According to Equation (5), the critical surface density in the presence of dynamical pressure can be written as

$$
\Sigma_{\mathrm{DP}}=\frac{\sigma^{2}}{\pi G H} e^{v_{S}^{2} / 4 \sigma^{2}} \approx \frac{\sigma \Omega}{\pi G} e^{v_{S}^{2} / 4 \sigma^{2}},
$$

letting the characteristic surface density of virialized clouds $\Sigma_{\text {vir }}=\sigma^{2} / \pi G H$ and using that the gas scale height $H$ is approximately $\sigma / \Omega$ near hydrostatic equilibrium in selfgravitating gas.

In Figure 14, we compare the radial profiles of this set of critical surface densities with the observed arm and interarm gas surface densities. As shown on the left, the shear critical surface density $\Sigma_{\text {shear }}$ falls below the observed surface density in the arm almost everywhere, suggesting that shear is not responsible for destroying clouds in the arm, consistent with the argument of, e.g., Elmegreen et al. (2003). The reverse appears to be true in the interarm where, as considered by Colombo et al. (2013a), the reduced cloud number density (compared with the arm) reflects an efficient cloud destruction mechanism.

In the zone of the bar, the shear and Toomre critical surface densities approach and even exceed the observed surface density, lending support to the idea that Coriolis forces plus shear (and shocks) in the bar can stabilize clouds and prevent subsequent SF. However, several lines of evidence suggest that cloud stabilization through this more traditional shear/shock scenario in the bar is weak and likely ineffective: (1) the bar itself is weak, as indicated by its small size and oval shape; (2) there is 


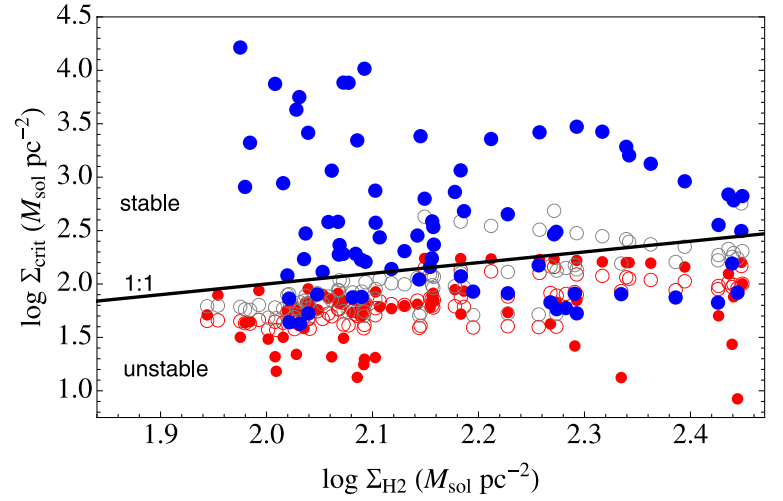

Figure 15. Critical surface densities versus $\Sigma_{\mathrm{H}_{2}}$ in the bar and spiral arms where different symbols and colors depict the potential for stabilization against gas self-gravity via Coriolis forces (the so-called Toomre critical surface density $\Sigma_{\text {toomre }}$; gray open symbols $)$, shear due to differential rotation $\left(\Sigma_{\text {shear }}\right.$; open red $)$, and including non-circular motions $\left(\Sigma_{\text {shear,sp }}\right.$; solid red) and dynamical pressure (solid blue). Measurements are extracted in 1".5 bins from the radial profiles in Figure 14. The solid black line shows the line of stability.

little evidence for a clearly defined pair of dust lanes running along the length of the bar (traced by either molecular gas or optical extinction), which are characteristic of a strong bar shock; and (3) as shown on the right of Figure 14, the threshold for stabilization through dynamical pressure is higher than these other thresholds, exceeding $\Sigma_{\text {gas }}$ by as much as an order of magnitude. Indeed, the critical surface density for dynamical pressure as expressed in Equation (D4) will always exceed the traditional Toomre critical surface density where $v_{S}>2 \sigma \sqrt{\ln \delta}$ for $\delta=\kappa / \Omega$ (i.e., $\delta=\sqrt{2}$, 1 , or 2 for rising, flat, or Keplerian rotation curves, respectively.)

According to Figure 14, it appears that SF in the bar zone may be suppressed and related most directly to stabilization through dynamical pressure. Stabilization via dynamical pressure is also evidently the strongest (if not the only) interpretation for the suppression of SF along specific portions of spiral arms. Only $\Sigma_{\mathrm{DP}}$ exceeds the observed arm surface densities at radii $35^{\prime \prime} \lesssim R \lesssim 60^{\prime \prime}$, where gas depletion times are measured to be longest (Figure 1), as well as at larger radii (i.e., $R \sim 80^{\prime \prime}$ and $R \sim 100^{\prime \prime}$, where $\tau_{\text {dep }}$ is also high.) As plotted in Figure 15, dynamical pressure is capable of supplying stability where other mechanisms fall short.

Note that even with adjustments to the empirical calibration factors $\alpha$ and $\alpha_{A}$, cloud stabilization through Coriolis forces and shear cannot account for the differences in SF properties between neighboring radial zones. Only dynamical pressure entails a non-monotonic radial variation in gas stability, given the pattern of streaming motions.

\section{REFERENCES}

Aalto, E., Hüttemeister, S., Scoville, N. Z., Thaddeus, P., et al. 1999, ApJ, $522,165 \mathrm{~A}$

Athanassoula, E. 1992, MNRAS, 259, 345

Ballesteros-Paredes, J., Vazquez-Semandeni, E., \& Scale, J. 1999, ApJ, 515,286

Barnes, E., \& Sellwood, J. 2003, AJ, 125, 1164

Barnes, E., Sellwood, J., \& Kosowsky, A. 2004, AJ, 128, 2724

Bigiel, F., Leroy, A., Walter, F., et al. 2008, AJ, 136, 2846

Binney, J., Gerhard, O. E., Stark, A. A., Bally, J., \& Uchida, K. I. 1991, MNRAS, 252,210

Binney, J., \& Tremaine, S. 1987, Galactic Dynamics (Princeton, NJ: Princeton Univ. Press)

Blitz, L., \& Rosolowsky, E. 2004, ApJ, 612, 29
Bonnell, I. A., Dobbs, C. L., Robitaille, T. R., \& Pringle, J. E. 2006, MNRAS, 365,37

Braine, J., Gratier, P., Kramer, C., et al. 2011, A\&A, 520, 107

Buta, R., \& Combes, F. 1996, FCPh, 17, 95

Calzetti, D., Kennicutt, R. C., Engelbracht, C. W., et al. 2007, ApJ, 666, 870

Ciardullo, R., Feldmeier, J. J., Jacoby, G. H., et al. 2002, ApJ, 577, 31

Colombo, D., Schinnerer, E., Hughes, A., et al. 2013a, ApJ, submitted

Colombo, D., Schinnerer, E., Meidt, S. E., et al. 2013b, ApJ, submitted

Combes, F., Garca-Burillo, S., Braine, J., et al. 2013, A\&A, 550, A41

Comeron, S., Martinez-Valpuesta, I., Knapen, J., \& Beckman, J. 2009, ApJL, 706, L256

Daddi, E., Elbaz, D., Walter, F., et al. 2010, ApJL, 714, L118

Debattista, V. P., Mayer, L., Carollo, C. M., et al. 2006, ApJ, 645, 209

de Blok, W. J. G., \& Walter, F. 2006, AJ, 131, 363

Dib, S., Helou, G., Moore, T. J. T., Urquhart, J. S., \& Dariush, A. 2012, ApJ, 758,125

Dobbs, C. L. 2008, MNRAS, 391, 844

Dobbs, C. L., \& Bonnell, I. A. 2007, MNRAS, 376, 1747

Dobbs, C. L., Burkert, A., \& Pringle, J. E. 2011, MNRAS, 417, 1318

Dumas, G., Schinnerer, E., \& Mundell, C. G. 2010, ApJ, 721, 911

Dumas, G., Schinnerer, E., Tabatabaei, F. S., et al. 2011, AJ, 141, 41

Egusa, F., Kohno, K., Sofue, Y., Nakanishi, H., \& Komugi, S. 2009, ApJ, 697,1870

Elmegreen, B. G. 1987, ApJ, 312, 626

Elmegreen, B. 1993, in Star Formation, Galaxies, and the Interstellar Medium, ed. J. Franco et al. (Cambridge: Cambridge Univ. Press), 337

Elmegreen, B. G. 1994, ApJ, 433, 39

Elmegreen, B. G., \& Elmegreen, D. M. 1986, ApJ, 311, 554

Elmegreen, B. G., Elmegreen, D. M., \& Leitner, S. M. 2003, ApJ, 590, 271

Eskew, M., Zaritsky, D., \& Meidt, S. E. 2012, AJ, 143, 139

Faber, S. M., \& Gallagher, J. S. 1979, ARA\&A, 17, 135

Feldmann, R., Gnedin, N. Y., \& Kravtsov, A. V. 2011, ApJ, 732, 115

Foyle, K., Rix, H.-W., Dobbs, C. L., Leroy, A. K., \& Walter, F. 2011, ApJ, 735,101

Garcia-Burillo, S., Combes, F., \& Gerin, M. 1993, A\&A, 274, 148

Garcia-Burillo, S., Combes, F., Schinnerer, E., Boone, F., \& Hunt, L. K. 2005, A\&A, 441, 1011

Garcia-Burillo, S., Fernandez-Garcia, S., Combes, F., et al. 2009, A\&A, 496, 85

Garcia-Burillo, S., Usero, A., Alonso-Herrero, A., et al. 2012, A\&A, 539, A8

Genzel, R., Tacconi, L. J., Gracia-Carpio, J., et al. 2010, MNRAS, 407, 2091

Gittins, D. M., \& Clarke, C. J. 2004, MNRAS, 349, 909

Gittins, D. M., Clarke, C. J., \& Bate, M. R. 2003, MNRAS, 340, 841

Groves, B., Krause, O., Sandstrom, K., et al. 2012, MNRAS, 426, 892

Gutierrez, L., Beckman, J. E., \& Buenrostro, V. 2011, AJ, 141, 113

Haan, S., Schinnerer, E., Emsellem, E., et al. 2009, ApJ, 692, 1623

Heiderman, A., Evans, N. J., II, Allen, L. E., Huard, T., \& Heyer, M. 2010, ApJ, 723, 1019

Hitschfeld, M., Kramer, C., Schuster, K. F., Garcia-Burillo, S., \& Stutzki, J. 2009, A\&A, 495, 795

Hocuk, S., \& Spaans, M. 2011, A\&A, 536, 41

Hopkins, P., Quataert, E., \& Murray, N. 2012, MNRAS, 421, 3488

Hughes, A., Meidt, S. E., Colombo, D., et al. 2013a, ApJ, arXiv:1309.3453

Hughes, A., Meidt, S. E., Schinnerer, E., et al. 2013b, ApJ, arXiv:1304.1209

Kenney, J., Carlstrom, J., \& Young, J. 1993, ApJ, 418, 687

Kennicutt, R. 1981, AJ, 86, 12

Kennicutt, R. C. 1989, ApJ, 344, 685

Kennicutt, R. C. 1998, ApJ, 498, 541

Kennicutt, R. C., Armus, L., Bendo, G., et al. 2003, PASP, 115, 928

Kennicutt, R. C., \& Evans, N. J. 2012, ARA\&A, 50, 531

Kim, C.-G., Kim, W.-T., \& Ostriker, E. C. 2008, ApJ, 681, 1148

Kim, W.-T., \& Ostriker, E. C. 2002, ApJ, 570, 132

Kim, W.-T., \& Ostriker, E. C. 2006, ApJ, 646, 213

Knapen, J., Beckman, J., Cepa, J., et al. 1992, ApJ, 385, 37

Krumholz, M., Dekel, A., \& McKee, C. F. 2012, ApJ, 745, 69

Krumholz, M., \& McKee, C. F. 2005, ApJ, 630, 250

Krumholz, M., McKee, C. F., \& Tumlinson, J. 2009, ApJ, 699, 850

Leroy, A. K., Bigiel, F., de Blok, W. J. G., et al. 2012, AJ, 144, 3

Leroy, A. K., Walter, F., Bigiel, F., et al. 2009, AJ, 137, 4670

Leroy, A. K., Walter, F., Brinks, E., et al. 2008, AJ, 136, 2782

Leroy, A. K., Walter, F., Sandstrom, K., et al. 2013, AJ, 146, 19

Longmore, S. N., Bally, J., Testi, L., et al. 2013, MNRAS, 429, 987

Luna, A., Bronfman, L., Carrasco, L., \& May, J. 2006, ApJ, 641, 938

Martig, M., Bournaud, F., Teyssier, R., \& Dekel, A. 2009, ApJ, 707,250

McKee, C. F., \& Ostriker, E. C. 2007, ARA\&A, 45, 565 
Meidt, S. E., Rand, R. J., \& Merrifield, M. R. 2009, ApJ, 702, 277

Meidt, S. E., Rand, R. J., Merrifield, M. R., Debattista, V. P., \& Shen, J. 2008a, ApJ, 676, 899

Meidt, S. E., Rand, R. J., Merrifield, M. R., Shetty, R., \& Vogel, S. 2008b, ApJ, 688,224

Meidt, S. E., Schinnerer, E., Knapen, J., et al. 2012, ApJ, 744, 17

Merrifield, M. R., Rand, R. J., \& Meidt, S. E. 2006, MNRAS, 366, 17

Minchev, I., Famaey, B., Combes, F., et al. 2011, A\&A, 527, 147

Mooney, T., \& Solomon, P. 1988, ApJL, 334, L51

Murray, N. 2011, ApJ, 729, 133

Murray, N., Quataert, E., \& Thompson, T. A. 2010, ApJ, 709, 191

Narayanan, D., Krumholz, M., Ostriker, E. C., \& Hernquist, L. 2011, MNRAS, 418, 664

Palunas, P., \& Williams, T. B. 2000, AJ, 120, 2884

Patrikeev, I., Fletcher, A., Stepanov, R., et al. 2006, A\&A, 458, 441

Pety, J., Schinnerer, E., Hughes, A., et al. 2013, ApJ, arXiv:1304.1396

Press, W. H., Teukolsky, S. A., Vetterling, W. T., \& Flannery, B. P. 1992, Numerical Recipes in FORTRAN (2nd ed.; Cambridge: Cambridge Univ. Press)

Rand, R. J. 1993, ApJ, 410, 68

Rautiainen, P., \& Salo, H. 1999, A\&A, 348, 737

Renaud, F., Kraljic, K., \& Bournaud, F. 2012, ApJL, 760, L16

Roberts, W., \& Stewart, G. 1987, ApJ, 314, 10

Rosolowsky, E. 2005, PASP, 117, 1403

Rosolowsky, E., \& Blitz, L. 2005, ApJ, 623, 826

Saintonge, A., Tacconi, L. J., Fabello, S., et al. 2012, ApJ, 758, 73

Sandstrom, K., Leroy, A. K., Walter, F., et al. 2013, ApJ, 777, 5
Schaye, J. 2004, ApJ, 609, 667

Schinnerer, E., Meidt, S. E., Colombo, D., et al. 2013, ApJ, arXiv:1304.1801

Schmidt, M. 1959, ApJ, 129, 243

Schruba, A., Leroy, A. K., Walter, F., et al. 2011, AJ, 142, 37

Schruba, A., Leroy, A. K., Walter, F., et al. 2012, AJ, 143, 138

Schuster, K. F., Kramer, C., Hitschfeld, M., Garcia-Burillo, S., \& Mookerjea, B. 2007, A\&A, 461, 143S

Seigar, M. S. 2005, MNRAS, 361, L20

Sheth, K., Regan, M., Hinz, J., et al. 2011, PASP, 122, 1397

Sheth, K., Vogel, S., Regan, M., et al. 2005, ApJ, 632, 217

Shetty, R., Glover, S. C., Dullemond, C. P., et al. 2011, MNRAS, 415, 3253

Shetty, R., Vogel, S. N., Ostriker, E. C., \& Teuben, P. J. 2007, ApJ, 665, 1138

Sygnet, J. F., Tagger, M., Athanassoula, E., \& Pellat, R. 1988, MNRAS, 232,733

Tagger, M., Sygnet, J. F., Athanassoula, E., \& Pellat, R. 1987, ApJL, 318, L43

Tan, J. C. 2010, ApJL, 710, L88

Toomre, A. 1964, ApJ, 139, 1217

Tully, R. B. 1974, ApJS, 27, 449

van der Laan, T., Schinnerer, E., Boone, F., et al. 2011, A\&A, 529, 45

Vogel, S. N., Rand, R. J., Gruendel, R. A., \& Teuben, P. J. 1993, PASP, 105, 60 Wada, K. 2008, ApJ, 675, 188

Walter, F., Brinks, E., de Blok, W. J. G., et al. 2008, AJ, 136, 2563

Weidner, C., Bonnell, I. A., \& Zinnecker, H. 2010, ApJ, 724, 1503

Zaritsky, D., Rix, H.-W., \& Rieke, M. 1993, Natur, 364, 313

Zhang, Z., \& Buta, R. 2012, arXiv:1203.5334

Zurita, A., Relano, M., Beckman, J. E., \& Knapen, J. H. 2004, A\&A, 413,73 